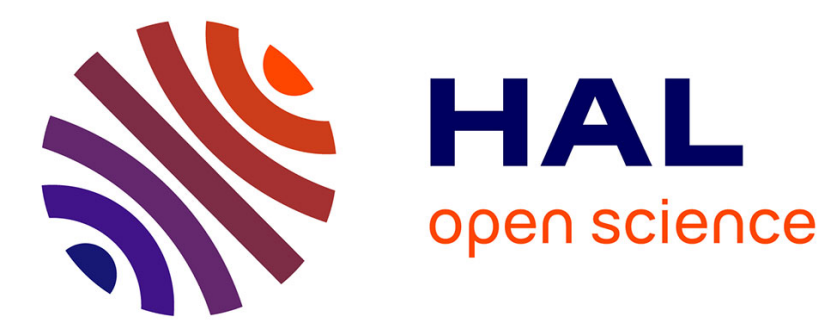

\title{
Nonlinear guidance and fuzzy control for three-dimensional path following of an underactuated autonomous underwater vehicle
}

Caoyang Yu, Xianbo Xiang, Lionel Lapierre, Qin Zhang

\section{To cite this version:}

Caoyang Yu, Xianbo Xiang, Lionel Lapierre, Qin Zhang. Nonlinear guidance and fuzzy control for three-dimensional path following of an underactuated autonomous underwater vehicle. Ocean Engineering, 2017, 146, pp.457-467. 10.1016/j.oceaneng.2017.10.001 • lirmm-01904325

\section{HAL Id: lirmm-01904325 \\ https://hal-lirmm.ccsd.cnrs.fr/lirmm-01904325}

Submitted on 24 Oct 2018

HAL is a multi-disciplinary open access archive for the deposit and dissemination of scientific research documents, whether they are published or not. The documents may come from teaching and research institutions in France or abroad, or from public or private research centers.
L'archive ouverte pluridisciplinaire HAL, est destinée au dépôt et à la diffusion de documents scientifiques de niveau recherche, publiés ou non, émanant des établissements d'enseignement et de recherche français ou étrangers, des laboratoires publics ou privés. 


\title{
Nonlinear guidance and fuzzy control for three-dimensional path following of an underactuated autonomous underwater vehicle
}

\author{
Caoyang $\mathrm{Yu}^{\mathrm{a}}$, Xianbo Xiang ${ }^{\mathrm{a}, *}$, Lionel Lapierre ${ }^{\mathrm{b}}$, Qin Zhang ${ }^{\mathrm{c}}$ \\ ${ }^{a}$ School of Naval Architecture and Ocean Engineering, Huazhong University of Science and Technology, 1037, Luoyu Road, Wuhan 430074, China \\ ${ }^{\mathrm{b}}$ Department of Robotics, CNRS-LIRMM-UMII, UMR 5506-CC 477, 161 rue Ada, 34392 Montpellier Cedex 5, France \\ ${ }^{\mathrm{c}}$ State Key Lab of Digital Manufacturing, Equipment and Technology, Huazhong University of Science and Technology, Wuhan 430074, China
}

\section{A R T I C L E I N F O}

\section{Keywords:}

Autonomous underwater vehicles

Path following

Guidance and control

Nonlinear fuzzy control

Sensitivity analysis

\begin{abstract}
A B S T R A C T
This paper proposes a simplified nonlinear fuzzy controller integrating an improved three-dimensional (3D) guidance law, in order to address the problem of path following for an underactuated autonomous underwater vehicle (AUV) exposed to unknown environmental disturbances. First, an improved 3D line-of-sight guidance law that makes full use of the essentially equivalent coordinate transformation is derived to transform 3D path following position errors into controlled guidance speeds, which also reduces the path following system form second-order to first-order. The side-slip angle and angle of attack are integrated into 3D guidance design to account for the underactuated configuration in sway and heave. Second, a nonlinear single-input fuzzy controller is designed to reduce computation complexity resulting from square rules in a double-input fuzzy controller, and to force the steerable speeds of an AUV to attain their guidance profiles. Subsequently, sensitivity analysis is adopted to suggest that the nonlinear fuzzy design with the convergent distribution and small slope for the single input have better robustness against unknown disturbances than the linear design. Finally, numerical examples with quantitative comparison are provided to illustrate the performance of the nonlinear single-input fuzzy controller for 3D path following of an underactuated AUV exposed to unknown environmental disturbances.
\end{abstract}

\section{Introduction}

Autonomous underwater vehicles (AUVs) are increasingly being used in the scientific, commercial, military, and policy sectors, such as underwater intervention (Zhang et al., 2015), monitoring and inspection (Xiang et al., 2010), target tracking (Shojaei and Dolatshahi, 2017), and sampling and patrolling (Zhang et al., 2007; Xiang et al., 2015a), etc. In order to accomplish diverse underwater tasks, it is desirable to automatically steer an AUV along a predefined path. Yet, most of AUVs equipped with two pairs of rudders and a stern propeller, such as REMUS 6000 and HUGIN 1000, cannot independently generate lateral and vertical control forces, which belong to a kind of underactuated systems with more degrees of freedom to be controlled than the number of independent control inputs and suffer from non-integrable second-order nonholonomic constraints (Yi et al., 2016; Xiang et al., 2016a). In addition, the AUV itself has the highly coupled dynamics and positive buoyancy (Zhu et al., 2017; Tanakitkorn et al., 2017), and it is persistently subjected to drift effects included by unpredictable environmental disturbances including the wind, waves and currents (Liang et al., 2017; Gao et al., 2016; Peng et al., 2015; Miao et al., 2017a). Hence, the motion control of an underactuated AUV is a difficult task and attracts the attention of numerous researchers worldwide (Xiang et al., 2017c).

The basic motion tasks of autonomous vehicles including mobile wheeled robots (MWRs), unmanned surface vehicles (USVs), remotely operated vehicles (ROVs) and AUVs are classified as trajectory tracking and path following (Do, 2016; Fossen et al., 2015; Wang et al., 2017a; Zhang et al., 2013; Chu et al., 2017b). In the trajectory tracking task, the vehicle must reach a specific point at a pre-assigned time instant. Hence, it inherently integrates space and time assignments into single assignment (Chu et al., 2017a; Qiao and Zhang, 2017; Wang et al., 2015, 2017b; Xiang et al., 2017d). While path following is to ensure the vehicle to reach and follow a desired Cartesian path, starting from a given initial configuration. For this task, it involves the separate construction of the geometric path and the dynamic assignment, namely emphasizing spatial convergence as a primary task, while considering the dynamic aspect to be of secondary importance. In this sense, path following represents a more flexible alternative compared to trajectory tracking (Breivik and Fossen, 2005a; Zheng et al., 2017a).

\footnotetext{
* Corresponding author.

E-mail address: xbxiang@hust.edu.cn (X. Xiang).
} 
There have been various efforts to develop intelligent controllers for path following control of an AUV in two-dimensional (2D) horizonal or vertical plane, such as feedback linearizing controller (Xiang et al., 2016b), backstepping controller (Lapierre and Soetanto, 2007; Xiang et al., 2015b), fuzzy controller (Guo et al., 2003), sliding mode controller (Chen et al., 2016), neural network controller (Peng et al., 2017a) and so on. These works consider unknown environmental disturbances, model uncertainty, and smooth transition of different configurations. Similar research methods can be found in path following and trajectory tracking control of MWRs, USVs and ROVs moving in the horizontal plane (Peng et al., 2013, 2017b; Chen and Tian, 2015; Wang et al., 2017c; Zheng et al., 2017b).

In fact, it is difficult to design an AUV path following controller from 2D decoupled control to three-dimensional (3D) coupled control (Cui et al., 2016; Zheng et al., 2017c; Xiang et al., 2017a). To the best of our knowledge, there have been few studies concerning 3D path following problem for an underactuated AUV exposed to environmental disturbances. A nonlinear 3D path following controller for an underactuated AUV was first proposed in (Encarnacao and Pascoal, 2000), while the singularity existed. This limitation was partially removed by introducing a virtual target point on the path in (Breivik and Fossen, 2005b), where another contribution was to first define line-of-sight (LOS) guidance angles in 3D space in order to achieve path following. Subsequently, many researchers followed and improved this LOS guidance law in kinematics and adopt multifarious intelligent controllers in dynamics to achieve path following control (Caharija et al., 2016; Miao et al., 2017b; Peymani and Fossen, 2015). More precisely, in Peymani and Fossen (2015), the virtually constrained motion control method was introduced and the fundamental principles of Lagrange mechanics were used to derive control laws. In Caharija et al. (2016), a cascaded framework composed of integral LOS guidance and a feedback linearizing proportional-derivative controller was developed for underactuated marine vehicles. Different from Breivik and Fossen (2005b), Aguiar built the tracking error in the body-fixed frame and adopted supervisory adaptive control and a nonlinear Lyapunov-based tracking control law to steer an underactuated AUV along a desired helix path (Aguiar et al, 2007). In addition, Do adopted Lyapunov's direct method, backstepping and parameter projection techniques to force an underactuated AUV to follow a predefined path despite of environmental disturbances and model uncertainty (Do et al., 2004). Yet, these controllers required substantial computational time and power due to the relatively complex derivations and decision making process.

As mentioned, some intelligent controllers require substantial computational time and power, which may not be applicable for the actual onboard controller of an AUV. In addition, a desired surge speed is often given in classic 3D path following for an underactuated AUV (Aguiar et al, 2007; Breivik and Fossen, 2005b; Caharija et al., 2016; Do et al., 2004; Peymani and Fossen, 2015), which results in the failure in determining the desired resultant speed due to drift effects in sway and heave. Motivated by those considerations, this paper releases the restriction on the given speed in 3D path following, and designs a simplified nonlinear single-input fuzzy controller with small calculation to achieve robust 3D path following. More specifically, the main contributions are enumerated as:

1. In order to solve the problem of the underactuated configuration in heave and sway, a novel 3D LOS guidance law is proposed to transform 3D path following position and orientation errors into controlled guidance speeds, which also reduces the dynamics following system form second-order to first-order. Different from Breivik and Fossen (2005a, 2005b), Miao et al. (2017b), the proposed LOS guidance law for $3 \mathrm{D}$ path following is based on the equivalent coordinate transformation among the resultant speed frame, body-fixed frame and inertial frame, which rigorously represents the relationship between the pitch, yaw, azimuth and elevation angles.

2. Compared to the double-input fuzzy controller (Guo et al., 2003; Xiang et al., 2017a), a single-input fuzzy path following controller is proposed in the dynamics layer. As an extension of planar motion (Ishaque et al., 2010, 2011), the single-input fuzzy controller is redesigned based on nonlinear technique to address the problem of 3D coupled motion with unknown environmental disturbances. In addition, the tracking errors of the AUV system under the nonlinear singleinput fuzzy controller are proved to be uniformly ultimately bounded.

3. The sensitivity analysis is adopted to suggest that the nonlinear single-input fuzzy design with convergent membership function distribution and small slope for the single input have better robustness against environmental disturbances than the linear single-input fuzzy design, and then numerical simulation validates the result of the sensitivity analysis as well as the robust performance of this nonlinear single-input fuzzy controller in 3D path following of an underactuated AUV exposed to unknown environmental disturbances.

The rest of this paper is organized as follows: Problem statement of 3D path following for an underactuated AUV is presented in Section 2. In Section 3, the improved 3D guidance law in kinematics is designed for an underactuated AUV. Subsequently, a simplified nonlinear single-input fuzzy controller is proposed in dynamics. Numerical simulation results are given in Section 4 to illustrate the robust performance. Section 5 contains some remarks and discussions.

\section{Problem statement}

This section describes the modeling of an underactuated AUV moving in 3D space and formulates the problem of 3D path following control.

\subsection{AUV modeling}

For an AUV in 3D space, the general kinematics model can be described by six motion components in surge, sway, heave, roll, pitch, and yaw directions. Due to the existence of a metacentric height between centers of gravity and buoyancy which can generate an enough large restoring moment to passively stabilize the roll oscillation, neglecting the roll motion dynamics is a common assumption for slender-body AUVs, i.e., the roll angle $\phi=0$ and the roll rate $p=0$ (Miao et al., 2017b; Xiang et al., 2017a). According to Do and Pan (2009), the kinematics equations of a five-degrees-of-freedom AUV are

$$
\left\{\begin{array}{l}
\dot{x}=\cos (\theta) \cos (\psi) u-\sin (\psi) v+\sin (\theta) \cos (\psi) w \\
\dot{y}=\cos (\theta) \sin (\psi) u+\cos (\psi) v+\sin (\theta) \sin (\psi) w \\
\dot{z}=-\sin (\theta) u+\cos (\theta) w \\
\dot{\theta}=q \\
\dot{\psi}=r / \cos (\theta)
\end{array}\right.
$$

where $x, y, z$ are coordinates of the origin of the body-fixed frame $\{B\}$ of the AUV expressed in the inertial frame $\{I\}$, and $\theta$ and $\psi$ define its pitch angle and yaw angle, respectively. $u, v, w, q$ and $r$ denote its body-fixed linear and angular speeds.

According to Do and Pan (2009), the dynamics equations of a five-degrees-of-freedom AUV can be simplified as

$$
\left\{\begin{array}{l}
\dot{u}=\frac{m_{22}}{m_{11}} v r-\frac{m_{33}}{m_{11}} w q-\frac{d_{11}}{m_{11}} u+\frac{\tau_{u}}{m_{11}}+\frac{\tau_{E u}}{m_{11}} \\
\dot{v}=-\frac{m_{11}}{m_{22}} u r-\frac{d_{22}}{m_{22}} v+\frac{\tau_{E v}}{m_{22}} \\
\dot{w}=\frac{m_{11}}{m_{33}} u q-\frac{d_{33}}{m_{33}} w+\frac{\tau_{E w}}{m_{33}} \\
\dot{q}=\frac{m_{33}-m_{11}}{m_{55}} u w-\frac{d_{55}}{m_{55}} q-\frac{G h \sin (\theta)}{m_{55}}+\frac{\tau_{q}}{m_{55}}+\frac{\tau_{E q}}{m_{55}} \\
\dot{r}=\frac{m_{11}-m_{22}}{m_{66}} u v-\frac{d_{66}}{m_{66}} r+\frac{\tau_{r}}{m_{66}}+\frac{\tau_{E r}}{m_{66}}
\end{array}\right.
$$

where $m_{(\cdot)}$ express system inertia coefficients, $d_{(\cdot)}$ denote hydrodynamic 
damping efforts, and $h=z_{g}-z_{b}$ with $z_{g}$ and $z_{b}$ denoting $z$-axis coordinates of centers of gravity $G$ and buoyancy. In addition, $\tau_{u}, \tau_{q}$ and $\tau_{r}$ are control inputs generated by the propeller and rudders in surge, pitch and yaw, respectively. $\tau_{E u}, \tau_{E v}, \tau_{E w}, \tau_{E q}$ and $\tau_{E r}$ are unknown environmental disturbances acting on the body-fixed frame of the AUV.

Remark 1. Since there are no lateral and vertical control forces, this five-degrees-of-freedom AUV is underactuated in sway and heave. Therefore, we cannot directly reduce the position error of 3D path following by adjusting heave and sway forces like a fully-actuated AUV.

Assumption 1. The unknown environmental disturbances are bounded, namely, $\left|\tau_{E u}\right| \leq \tau_{E u \max },\left|\tau_{E v}\right| \leq \tau_{E v \max },\left|\tau_{E w}\right| \leq \tau_{E w \max },\left|\tau_{E q}\right| \leq \tau_{E q \max }$, and $\left|\tau_{E r}\right| \leq \tau_{E r \max }$ where $\tau_{E u \max }, \tau_{E v \max }, \tau_{E w \max }, \tau_{E q \max }$, and $\tau_{E r \max }$ are unknown positive constants, and $|\cdot|$ denotes the absolute value of a variable (Fossen and Lekkas, 2017; Miao et al., 2017a).

\subsection{Problem formulation}

As depicted in Fig. 1, an underactuated AUV represented by point $Q$ follows a desired 3D Cartesian path continuously parameterized by a scalar variable $\varpi \in \mathbb{R}$. Note that the evolution of $\varpi$ is time-independent. Denote inertial position and speed vectors of a virtual particle $P$ on the path by $\quad \boldsymbol{p}_{P}(\varpi)=\left[x_{P}(\varpi), y_{P}(\varpi), z_{P}(\varpi)\right]^{\top} \quad$ and $\quad \dot{p}_{P}(\varpi)=$ $\left[x_{P}^{\prime}(\varpi) \dot{\varpi}, y_{P}^{\prime}(\varpi) \dot{\varpi}, z_{P}^{\prime}(\varpi) \dot{\varpi}\right]^{\top} \quad$ with $\quad x_{P}^{\prime}(\varpi)=\frac{\partial x_{P}}{\partial \varpi}, y_{P}^{\prime}(\varpi)=\frac{\partial y_{P}}{\partial \varpi}, \quad$ and $z_{P}^{\prime}(\varpi)=\frac{\partial z_{p}}{\partial \varpi}$, respectively. The resultant speed vector of the AUV described in the wind frame $\{W\}$ is $\boldsymbol{v}_{W}=[U, 0,0]^{\top}$ with $\left\|\boldsymbol{v}_{W}\right\|=U=\sqrt{u^{2}+v^{2}+w^{2}}$, where $\|\cdot\|$ denotes the Euclidean norm of a vector.

Let the spatial vector $\varepsilon=\overrightarrow{P Q}$ between the virtual particle $P$ and the point $Q$ represent the path following position error. Then, the problem of 3D path following control for an underactuated AUV can be formulated as follows:

Given a desired 3D path parameterized by a particle $P$, develop dynamics control laws for control force and moments of an underactuated AUV in the presence of unknown environmental disturbances such that the distance from particle $P$ to point $Q$ converges to a small region of the origin, and the AUV achieves a desired resultant speed $U_{d}$ while its resultant speed is basically aligned with the tangent of the path, namely

$\lim _{t \rightarrow \infty}\left(\|\boldsymbol{\varepsilon}\|, U_{d}-U, \chi_{P}-\chi, v_{P}-v\right)=\left(\delta_{1}, \delta_{2}, \delta_{3}, \delta_{4}\right)$

where $\delta_{i}(i=1,2,3,4)$ are small constants near zero, $\chi$ and $v$ denote the azimuth angle and elevation angle of the AUV, and $\chi_{P}$ and $v_{P}$ denote the azimuth angle and elevation angle of particle $P$ on the path. These four angles are described in Fig. 1 and $x_{M}$ is their auxiliary line linking the resultant speed to the inertial frame.

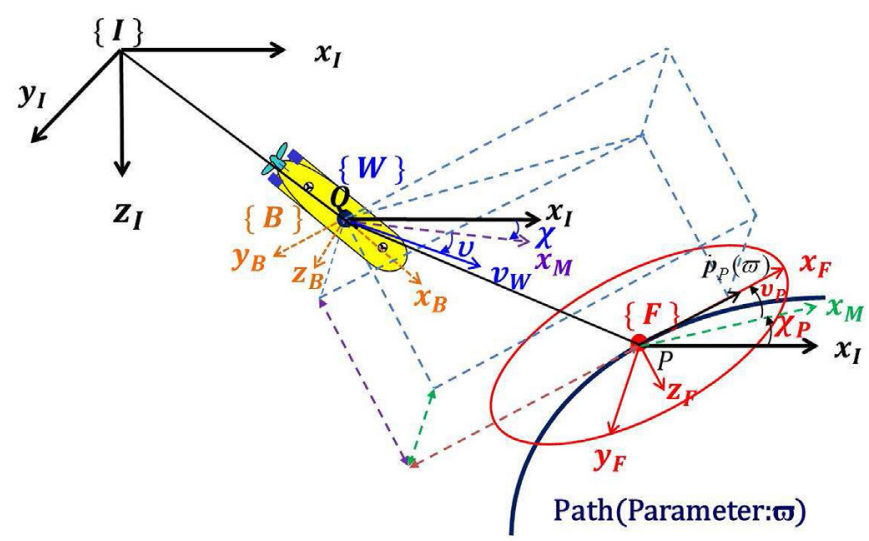

Fig. 1. Frame definitions of 3D path following.

\section{3D path following control design}

As shown in Fig. 2, an improved guidance law for 3D path following control of an underactuated AUV is presented in the kinematics layer, and then the nonlinear single-input fuzzy controller in the dynamics layer is designed to track desired guidance profiles generated by 3D guidance law.

\subsection{Improved $3 D$ guidance law in kinematics}

Associated with particle $P$, the corresponding path frame $\{F\}$ using the speed direction of particle $P$ as its $x$-axis can be built. As shown in Fig. 1, two consecutive coordinate rotations about the $z$-axis and $y$-axis of frame $\{I\}$ are required to align the $x$-axis of frame $\{I\}$ with that of frame $\{F\}$. Hence, the $y$-axis of frame $\{F\}$ is parallel to the $x y$-plane of frame $\{I\}$, and its z-axis is defined by the right-hand rule. Define two rotation angles $\chi_{P}$ and $v_{P}$ from frame $\{F\}$ to frame $\{I\}$ as

$$
\left\{\begin{array}{l}
\chi_{P}(\varpi)=\operatorname{atan} 2\left(y_{P}^{\prime}(\varpi), x_{P}^{\prime}(\varpi)\right) \\
v_{P}(\varpi)=\arctan \left(\frac{-z_{P}^{\prime}(\varpi)}{\sqrt{x_{P}^{\prime}(\varpi)^{2}+y_{P}^{\prime}(\varpi)^{2}}}\right)
\end{array}\right.
$$

where the function atan 2 is the arctangent function with two arguments which ensures the mapping $\chi_{P}(\varpi) \in(-\pi, \pi]$. And then, the rotation from $\boldsymbol{R}_{F}$ frame $\{F\}$ to frame $\{I\}$ can be denoted by

$\boldsymbol{R}_{F}=\boldsymbol{R}_{z}\left(\chi_{P}\right) \boldsymbol{R}_{y}\left(v_{P}\right)$

with $\quad \boldsymbol{R}_{z}\left(\chi_{P}\right)=\left[\cos \left(\chi_{P}\right),-\sin \left(\chi_{P}\right), 0 ; \sin \left(\chi_{P}\right), \cos \left(\chi_{P}\right), 0 ; 0,0,1\right] \quad$ and $\boldsymbol{R}_{y}\left(v_{P}\right)=\left[\cos \left(v_{P}\right), 0, \sin \left(v_{P}\right) ; 0,1,0 ;-\sin \left(v_{P}\right), 0, \cos \left(v_{P}\right)\right]$.

Consequently, the spatial position error vector $\varepsilon$ between particle $P$ and point $Q$ expressed in frame $\{F\}$ is given by

$\boldsymbol{\varepsilon}=\left[x_{e}, y_{e}, z_{e}\right]^{\top}=\boldsymbol{R}_{F}^{\top}\left(\boldsymbol{p}-\boldsymbol{p}_{P}\right)$

where $x_{e}, y_{e}$ and $z_{e}$ represent the along-track error, the cross-track error, and the vertical-track from particle $P$ to point $Q$ in frame $\{F\}$, respectively. $\|\boldsymbol{\varepsilon}\|=\sqrt{x_{e}^{2}+y_{e}^{2}+z_{e}^{2}}$ is the off-track error between them, as shown in Fig. 1. In addition, $\boldsymbol{p}=[x, y, z]^{\top}$ is the inertial position vector of the underactuated AUV in 3D space.

As the primary control objective of 3D path following in (3) is to reduce the off-track error, the following positive definite Lyapunov function candidate is considered

$V_{\boldsymbol{\varepsilon}}=\frac{1}{2}\|\boldsymbol{\varepsilon}\|^{2}$

Similarly to Breivik and Fossen (2005a), the derivative of (7) is

$$
\begin{aligned}
\dot{V}_{\varepsilon}= & x_{e}\left(U_{d} \cos \left(v_{r}\right) \cos \left(\chi_{r}\right)-U_{P}\right)-z_{e} U_{d} \sin \left(v_{r}\right) \\
& +y_{e} U_{d} \sin \left(\chi_{r}\right) \cos \left(v_{r}\right)
\end{aligned}
$$

where $\chi_{r}$ and $v_{r}$ are two rotation angles of the rotation $\boldsymbol{R}_{W_{d}}^{F}=$ $\boldsymbol{R}_{z}\left(\chi_{r}\right) \boldsymbol{R}_{\boldsymbol{y}}\left(v_{r}\right)$ from the desired flow frame $\left\{W_{d}\right\}$ of the AUV to frame $\{F\}$ as shown in Fig. 1, and $U_{P}=\left\|\dot{\boldsymbol{p}}_{P}(\varpi)\right\|$ is the size of the resultant speed of particle $P$.

In order to drive the derivative of $V_{\varepsilon}$ negative, the resultant speed of particle $P$ can be assigned to evolve as

$U_{p}=U_{d} \cos \left(\chi_{r}\right) \cos \left(v_{r}\right)+k_{x} x_{e}$

with the positive speed control gain $k_{x}$. Since $U_{P}=\left\|\dot{\boldsymbol{p}}_{P}(\varpi)\right\|$ and $\left\|\dot{\boldsymbol{p}}_{P}(\varpi)\right\|=\sqrt{x_{P}^{\prime}(\varpi)^{2}+y_{P}^{\prime}(\varpi)^{2}+z_{P}^{\prime}(\varpi)^{2}} \dot{\varpi}$, the rate of evolution for the path scalar variable $\varpi$ can be calculated by 


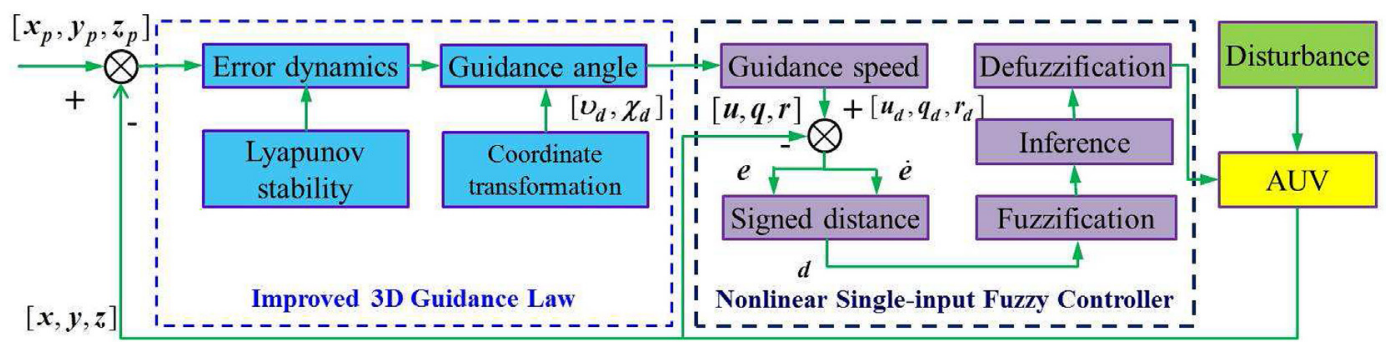

Fig. 2. Control framework of AUV path following.

$\dot{\varpi}=\frac{U_{d} \cos \left(\chi_{r}\right) \cos \left(v_{r}\right)+k_{x} x_{e}}{\sqrt{x_{P}^{\prime}(\varpi)^{2}+y_{P}^{\prime}(\varpi)^{2}+z_{P}^{\prime}(\varpi)^{2}}}$

Note that this virtual control law for particle $P$ on the path is nonsingular and can be easily achieved, which introduces an extra degree of freedom in the path following control design of an AUV.

According to the LOS guidance principles, two rotation angles of matrix $\boldsymbol{R}_{W_{d}}^{F}$ can be defined as

$\left\{\chi_{r}=k_{\chi} \tanh \left(-k_{y} y_{e} / \Delta_{y}\right)\right.$

$\left\{\begin{array}{l}x_{r}=k_{v} \tanh \left(k_{z} z_{e} / \Delta_{z}\right) \\ v_{2}\end{array}\right.$

where $k_{\chi} \in\left(0, \frac{\pi}{2}\right)$ and $k_{v} \in\left(0, \frac{\pi}{2}\right)$ are chosen to let $\chi_{r} \in\left(-\frac{\pi}{2}, \frac{\pi}{2}\right)$ and $v_{r} \in\left(-\frac{\pi}{2}, \frac{\pi}{2}\right)$. In addition, $k_{y}, k_{z}, \Delta_{y}$, and $\Delta_{z}$ are positive. Hence, $\dot{V}_{\varepsilon}=$ $-k_{x} x_{e}^{2}-\sin \left(k_{\chi} \tanh \left(\frac{k_{y}\left|y_{e}\right|}{\Delta_{y}}\right)\right) \cos \left(v_{r}\right)\left|y_{e}\right| U_{d}-\sin \left(k_{v} \tanh \left(\frac{k_{z}\left|z_{e}\right|}{\Delta_{z}}\right)\right)\left|z_{e}\right| U_{d}$ is negative definite, which indicates that the position error tends to zero under the control laws (10) and (11).

Similarly to the definition of $\boldsymbol{R}_{F}$, the rotation $\boldsymbol{R}_{W_{d}}$ from the desired wind frame $\left\{W_{d}\right\}$ of the AUV using the desired resultant speed direction as its $x$-axis to frame $\{I\}$ is defined as

$\boldsymbol{R}_{W_{d}}=\boldsymbol{R}_{z}\left(\chi_{d}\right) \boldsymbol{R}_{y}\left(v_{d}\right)$

where $\chi_{d}$ and $v_{d}$ denote the desired azimuth angle and elevation angle of the AUV, respectively.

It is concluded that both the rotation $\boldsymbol{R}_{W_{d}}$ and the consecutive rotations of $\boldsymbol{R}_{W_{d}}^{F}$ and $\boldsymbol{R}_{F}$ map the desired resultant speed vector $\boldsymbol{v}_{W_{d}}$ expressed in frame $\left\{W_{d}\right\}$ equivalently to frame $\{I\}$, which can be expressed as

$\boldsymbol{R}_{F} \boldsymbol{R}_{W_{d}}^{F} \boldsymbol{v}_{W_{d}}=\boldsymbol{R}_{W_{d}} \boldsymbol{v}_{W_{d}}$

with $\boldsymbol{v}_{W_{d}}=\left[U_{d}, 0,0\right]^{\top}$

Expanding (13) yields that

$\left\{v_{d}=\arcsin \left(\sin \left(v_{P}\right) \cos \left(v_{r}\right) \cos \left(\chi_{r}\right)+\cos \left(v_{P}\right) \sin \left(v_{r}\right)\right)\right.$

$\left\{\chi_{d}=\operatorname{atan} 2\left(\chi_{d y}, \chi_{d x}\right)\right.$

with $\quad \chi_{d y}=\sin \left(\chi_{P}\right) \cos \left(\chi_{r}\right) \cos \left(v_{P}\right) \cos \left(v_{r}\right)+\cos \left(\chi_{P}\right) \sin \left(\chi_{r}\right) \cos \left(v_{r}\right)-$ $\sin \left(v_{P}\right) \sin \left(v_{r}\right) \sin \left(\chi_{P}\right) \quad$ and $\quad \chi_{d x}=\cos \left(\chi_{P}\right) \cos \left(\chi_{r}\right) \cos \left(v_{P}\right) \quad \cos \left(v_{r}\right)-$ $\sin \left(\chi_{P}\right) \sin \left(\chi_{r}\right) \cos \left(v_{r}\right)-\cos \left(\chi_{P}\right) \sin \left(v_{P}\right) \sin \left(v_{r}\right)$.

According to $\boldsymbol{v}_{B}=\boldsymbol{R}_{W}^{B} \boldsymbol{v}_{W}$ (Fossen, 2011), the desired body-fixed linear speed vector $\boldsymbol{v}_{B_{d}}$ can be designed as

$\boldsymbol{v}_{B_{d}}=\boldsymbol{R}_{W}^{B} \boldsymbol{v}_{W_{d}}$

where $\quad \boldsymbol{v}_{B}=[u, v, w]^{\top}, \quad \boldsymbol{v}_{B_{d}}=\left[u_{d}, v_{d}, w_{d}\right]^{\top}, \quad \boldsymbol{R}_{W}^{B}=\boldsymbol{R}_{y}^{\top}(\alpha) \boldsymbol{R}_{z}^{\top}(-\beta) \quad$ with $\boldsymbol{R}_{y}(\alpha)=[\cos (\alpha), 0, \sin (\alpha) ; 0,1,0 ;-\sin (\alpha), 0, \cos (\alpha)] \quad$ and $\boldsymbol{R}_{z}(-\beta)=[\cos (\beta), \sin (\beta), 0 ;-\sin (\beta), \cos (\beta), 0 ; 0,0,1], \alpha$ and $\beta$ are the sideslip angle and angle of attack of the AUV.

Expanding (15) gives the desired surge speed $u_{d}$ as
$u_{d}=U_{d} \cos (\alpha) \cos (\beta)$

Remark 2. In classic path following of an underactuated AUV, the desired surge speed is given directly or assume that the surge speed always keeps constant due to underactuated configurations in sway and heave, while this paper extends to giving a desired resultant speed. By designing the desired surge speed as (16) based on the coordinate transformation from the resultant speed frame to the body-fixed frame and then deriving the surge speed control law, the resultant speed of an underactuated AUV can be guaranteed to attain its given profile. More specifically, if $u=u_{d}$ under the dynamics control law, $U=U_{d}$ owing to (16) and $u=U \cos (\alpha) \cos (\beta)$. In addition, we can obtain that $v_{d}=U_{d} \sin (\beta), \quad v=U \sin (\beta), \quad w_{d}=U_{d} \sin (\alpha) \cos (\beta) \quad$ and $w=U \sin (\alpha) \cos (\beta)$. Therefore, $v=v_{d}$ and $w=w_{d}$ in this case, which means that $\boldsymbol{v}_{B}=\boldsymbol{v}_{B_{d}}$ will be guaranteed when $u=u_{d}$.

Inspired by the solution of the desired azimuth angle $\chi_{d}$ and elevation angle $v_{d}$, the truth is that both the rotation $\boldsymbol{R}_{W_{d}}$ from frame $\left\{W_{d}\right\}$ to frame $\{I\}$ and the consecutive rotations from frame $\left\{W_{d}\right\}$ to the desired bodyfixed frame $\left\{B_{d}\right\}$ and then to frame $\{I\}$ map the desired resultant speed vector $\boldsymbol{v}_{W_{d}}$ equivalently to frame $\{I\}$, namely

$\boldsymbol{R}_{z}\left(\psi_{d}\right) \boldsymbol{R}_{y}\left(\theta_{d}\right) \boldsymbol{R}_{y}^{\top}(\alpha) \boldsymbol{R}_{z}^{\top}(-\beta) \boldsymbol{v}_{W_{d}}=\boldsymbol{R}_{z}\left(\chi_{d}\right) \boldsymbol{R}_{y}\left(v_{d}\right) \boldsymbol{v}_{W_{d}}$

Expanding (17) leads to the desired pitch angle $\theta_{d}$ and the desired yaw angle $\psi_{d}$ as

$\left\{\theta_{d}=\arcsin \left(\sin \left(v_{d}\right) / \cos (\beta)\right)+\alpha\right.$

$\left\{\psi_{d}=\operatorname{atan} 2\left(\psi_{d y}, \psi_{d x}\right)\right.$

with $\quad \psi_{d y}=\cos (\beta) \cos \left(\theta_{d}-\alpha\right) \sin \left(\chi_{d}\right) \cos \left(v_{d}\right)-\sin (\beta) \cos \left(\chi_{d}\right) \cos \left(v_{d}\right)$ and $\psi_{d x}=\sin (\beta) \sin \left(\chi_{d}\right) \cos \left(v_{d}\right)+\cos (\beta) \cos \left(\theta_{d}-\alpha\right) \cos \left(\chi_{d}\right) \cos \left(v_{d}\right)$.

Remark 3. The desired pitch angle $\theta_{d}$ and the desired yaw angle $\psi_{d}$ in this paper are different from $\theta_{d}=v_{d}-v+\theta$ and $\psi_{d}=\chi_{d}-\chi+\psi$ defined in (Breivik and Fossen, 2005b). In fact, the azimuth angle $\chi$ and the elevation angle $v$ of an AUV are defined as

$\left\{\begin{array}{l}\chi=\operatorname{atan} 2(\dot{y}, \dot{x}) \\ v=\arctan \left(-\dot{z} / \sqrt{\dot{x}^{2}+\dot{y}^{2}}\right)\end{array}\right.$

Substituting (1) into (19), it can be seen that $\chi=f_{\chi}(\theta, \psi)$ and $v=f_{v}(\theta)$, namely $\psi=f_{\psi}(\chi, v)$ and $\theta=f_{\theta}(v)$. Therefore, the definitions of $\theta_{d}$ and $\psi_{d}$ in (Breivik and Fossen, 2005b) might not be very rigorous, especially with regard to $\psi_{d}$. Note that if $\theta=\theta_{d}$ and $\psi=\psi_{d}$ under the dynamics control law in this paper, $v=v_{d}$ and $\chi=\chi_{d}$ will be guaranteed in case of $u=u_{d}$ owing to $\boldsymbol{R}_{z}(\chi) \boldsymbol{R}_{y}(v) \boldsymbol{v}_{W}=\boldsymbol{R}_{z}\left(\chi_{d}\right) \boldsymbol{R}_{y}\left(v_{d}\right) \boldsymbol{v}_{W}$ by combining (17) with $\boldsymbol{R}_{z}(\psi) \boldsymbol{R}_{y}(\theta) \boldsymbol{R}_{y}^{\top}(\alpha) \boldsymbol{R}_{z}^{\top}(-\beta) \boldsymbol{v}_{W}=\boldsymbol{R}_{z}(\chi) \boldsymbol{R}_{y}(v) \boldsymbol{v}_{W}$. Therefore, if $u$ is equal to $u_{d}$ that is updated by (16), $\theta$ and $\psi$ are equal to $\theta_{d}$ and $\psi_{d}$ that are updated by (18), the position error vector $\varepsilon$ will be rendered stable based on Lyapunov's direct method, and the resultant speed of the AUV will 
align with the tangent of the path.

\subsection{Nonlinear fuzzy controller in dynamics}

This subsection first designs a conventional double-input fuzzy controller to track the guidance profiles generated in the kinematics layer. Subsequently, the double-input fuzzy controller is simplified into a single-input fuzzy controller based on the signed distance method to reduce computation complexity. By introducing the asymmetry factor and slope for the single input, the output surface of the single-input fuzzy controller becomes nonlinear to account for complicated nonlinear AUV dynamics and unknown environmental disturbances. Finally, the sensitivity analysis preliminarily suggests that the nonlinear single-input fuzzy design have better robustness against environmental disturbances than the linear single-input fuzzy design.

\subsubsection{Double-input fuzzy controller design}

Based on the desired Euler angles $\theta_{d}$ and $\psi_{d}$ generated by the improved 3D guidance law, the angle tracking error $\boldsymbol{e}_{\text {ang }}$ can be defined as

$\boldsymbol{e}_{\text {ang }}=\left[e_{\theta}, e_{\psi}\right]^{\top}=\left[\theta_{d}-\theta, \psi_{d}-\psi\right]^{\top}$

Considering the model (1) and backstepping technique, the desired pitch speed $q_{d}$ and the desired yaw speed $r_{d}$ of the AUV can be designed as

$\left\{\begin{array}{l}q_{d}=\dot{\theta}_{d}+k_{\theta} e_{\theta} \\ r_{d}=\dot{\psi}_{d}+k_{\psi} e_{\psi}+\left(1-\frac{1}{\cos (\theta)}\right) r\end{array}\right.$

By resorting to the improved 3D guidance law, 3D path following control can be converted into tracking the desired linear and angular speeds, which reduces the dynamics following system form second-order to first-order. Hence, we define speed tracking error $\boldsymbol{e}_{\text {spe }}$ as follows

$\boldsymbol{e}_{s p e}=\left[e_{u}, e_{q}, e_{r}\right]^{\top}=\left[u_{d}-u, q_{d}-q, r_{d}-r\right]^{\top}$

In order to drive speed error $\boldsymbol{e}_{\text {spe }}$ to a small region of zero in the presence of unknown environmental disturbances, the nonlinear fuzzy logic technique is adopted to reject model uncertainties of the nonlinear dynamics system (Ishaque et al., 2011; Wang et al., 2016; Xiang et al., 2017b; Yu et al., 2017). In addition, the feedback linearizing technique is introduced to eliminate the system nonlinearity. Hence, the fuzzy-logicbased control laws for an underactuated AUV with the dynamics model (2) can be designed as

$\left\{\begin{array}{l}\tau_{u}=\tau_{u 0}+m_{11}\left(\dot{u}_{d}+\int_{0}^{t} k_{f u} f_{u}\left(k_{e u} e_{u}, k_{e u} \dot{e}_{u}\right) d \tau\right) \\ \tau_{q}=\tau_{q 0}+m_{55}\left(\dot{q}_{d}+\int_{0}^{t} k_{f q} f_{q}\left(k_{e q} e_{q}, k_{\dot{~} q} \dot{e}_{q}\right) d \tau\right) \\ \tau_{r}=\tau_{r 0}+m_{66}\left(\dot{r}_{d}+\int_{0}^{t} k_{f r} f_{r}\left(k_{e r} e_{r}, k_{\dot{~}} \dot{e}_{r}\right) d \tau\right)\end{array}\right.$

where $\quad \tau_{u 0}=-m_{22} v r+m_{33} w q+d_{11} u, \quad \tau_{q 0}=\left(m_{11}-m_{33}\right) u w+d_{55} q$ $+G h \sin (\theta), \tau_{r 0}=\left(m_{22}-m_{11}\right) u v+d_{66} r, k_{f u}, k_{f q}$ and $k_{f u}$ are scaling factors of outputs $f_{u}(\cdot), f_{q}(\cdot)$ and $f_{r}(\cdot)$ of the designed double-input fuzzy controller in surge, pitch and yaw, respectively. $k_{e^{*}}$ and $k_{e_{*}}$ denote scaling factors of original inputs $e_{*}$ and $\dot{e}_{*}$ with $* \in\{u, q, r\}$, respectively.

\subsubsection{Single-input fuzzy controller design}

The above double-input fuzzy controller is solved through three steps, i.e., fuzzification, fuzzy inference and defuzzification (Xiang et al.,2017a, Ju and Liu, 2011, 2012). This fuzzy controller has two scaling inputs, namely the error $\bar{e}_{*}=k_{e^{*}} e_{*}$ and derivative of error $\dot{\bar{e}}_{*}=k_{\dot{e}_{*}} \dot{e}_{*}$. The corresponding fuzzy subsets are defined as NB, NM, NS, ZE, PS, PM, and PB, and show symmetrical distribution of triangular membership functions. The adopted fuzzy rules can be created on a 2D phase-plane as shown in Fig. 3, where each point on the particular diagonal lines has the same magnitude that is proportional to the distance from it to the main diagonal line $L_{Z E}$.
By observing the consistent pattern of outputs in Fig. 3, it is possible to obtain the corresponding output using only a single-variable input instead of two-variable input. This reduction is known as the signed distance method (Choi et al., 2000). The single-input variable is named as $d$ that represents the absolute distance magnitude from the input point $\left(\bar{e}_{*}, \dot{e}_{*}\right)$ to the main diagonal line $L_{Z E}$. It is noted that the main diagonal line can be represented by the following straight line function

$\bar{e}_{*}+\lambda_{*} \dot{\bar{e}}_{*}=0$

where $\lambda_{*}$ is the slope of the straight line.

Hence, the distance $d_{*}$ from point $\left(\overline{\boldsymbol{e}}_{*}, \dot{\bar{e}}_{*}\right)$ to line $L_{Z E}$ is

$d_{*}=\frac{\bar{e}_{*}+\lambda_{*} \dot{\bar{e}}_{*}}{\sqrt{1+\lambda_{*}^{2}}}$.

Note that as the slope $\lambda_{*}$ becomes large, the sensitivity of the scaling error will decrease while the sensitivity of the scaling derivative of error will increase.

Compared with a 2D table required by the conventional double-input fuzzy controller, the derivation of distance input variable generates a simple look-up table depicted in Table 1 , where $L_{N B}, L_{N M}, L_{N S}, L_{P S}, L_{P M}$, and $L_{P B}$ are diagonal lines in Fig. 3 and NB, NM, NS, ZE, PS, PM, and PB represent the output of the corresponding diagonal line. Obviously, $49 \times 3$ rules required by the conventional double-input fuzzy controller reduce to $7 \times 3$ rules required by the simplified single-input fuzzy controller that is exclusively determined by $d_{*}$.

By adopting the single-input fuzzy control technique, the control laws (23) can be rewritten as

$\left\{\begin{array}{l}\tau_{u}=\tau_{u 0}+m_{11}\left(\dot{u}_{d}+\int_{0}^{t} k_{f u} f_{u}\left(d_{u}\right) d \tau\right) \\ \tau_{q}=\tau_{q 0}+m_{55}\left(\dot{q}_{d}+\int_{0}^{t} k_{f q} f_{q}\left(d_{q}\right) d \tau\right) \\ \tau_{r}=\tau_{r 0}+m_{66}\left(\dot{r}_{d}+\int_{0}^{t} k_{f r} f_{r}\left(d_{r}\right) d \tau\right)\end{array}\right.$

\subsubsection{Nonlinear single-input fuzzy controller design}

In the fuzzification of (26), the membership functions of the input $d_{*}$ have trapezoidal and triangular types as depicted in Fig. 4, and the corresponding universe of discourse is listed in Table 2, where $\sigma \in\left(-\frac{1+\lambda}{6 \sqrt{1+\lambda^{2}}}, \frac{1+\lambda}{6 \sqrt{1+\lambda^{2}}}\right)$ represents the asymmetry factor, trapmf denotes the trapezoidal membership function and trimf denotes the triangular membership function. As shown in Fig. 4, $\sigma=0$ means that the membership functions show the symmetric distribution, $\sigma>0$ means that the membership functions show the convergent distribution, and $\sigma<0$ means that the membership functions show the divergent distribution.

For the membership function of the output, the general first-order Takagi-Sugeno-Kang method instead of Mamdani method is chosen. Hence, the membership function value $l_{i}$ of the output in each rule is a

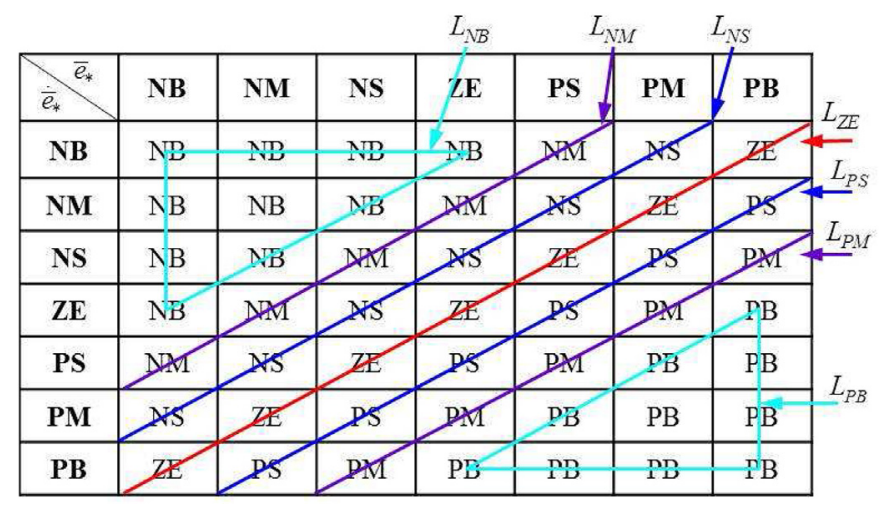

Fig. 3. Fuzzy rules with the Toeplitz structure. 
Table 1

Single-input fuzzy control rules for 3D path following.

\begin{tabular}{llllllll}
\hline$i$ & 1 & 2 & 3 & 4 & 5 & 6 & 7 \\
\hline$d_{*}$ & $L_{N B}$ & $L_{N M}$ & $L_{N S}$ & $L_{Z E}$ & $L_{P S}$ & $L_{P M}$ & $L_{P B}$ \\
$l_{i}$ & $\mathrm{NB}$ & $\mathrm{NM}$ & $\mathrm{NS}$ & $\mathrm{ZE}$ & $\mathrm{PS}$ & $\mathrm{PM}$ & $\mathrm{PB}$ \\
\hline
\end{tabular}

linear function with respect to the input $d_{*}$ as

$l_{i}=a_{i} d_{*}+b_{i}$

where $a_{i}$ and $b_{i}$ are the slope and intercept of the output line, respectively. In the fuzzy inference, $l_{i}$ is multiplied by the respective membership degree value to contribute to the fuzzy output of each rule.

Subsequently, choose Table 1 as the inference rule. Using the center of gravity operator, the output of defuzzification can be calculated as

$f_{*}=\frac{\sum_{i=1}^{7} \mu_{i} l_{i}}{\sum_{i=1}^{7} \mu_{i}}$

where $\mu_{i}$ is the membership degree value of the $i$-th rule.

As shown in Fig. 4, there are only two non-zero membership degree values $\mu_{k}$ and $\mu_{k+1}$ for $d_{*} \in\left(x_{1}, x_{7}\right)$ with $k \in[1,6]$ and there is only one non-zero membership degree value $\mu_{k} \equiv 1$ for $d_{*} \notin\left(x_{1}, x_{7}\right)$ with $k \in\{1,7\}$, namely,

$$
\sum_{i=1}^{7} \mu_{i}=\left\{\begin{array}{cc}
1, & d_{*} \notin\left(x_{1}, x_{7}\right) \\
\frac{x_{k+1}-d_{*}}{x_{k+1}-x_{k}}+\frac{d_{*}-x_{k}}{x_{k+1}-x_{k}}, & d_{*} \in\left(x_{1}, x_{7}\right) \\
=1, d_{*} \in \mathbb{R} &
\end{array}\right.
$$

On the one hand, when $d_{*} \in\left(x_{1}, x_{7}\right)$, the output (28) can be rewritten as

$$
\begin{aligned}
f_{*}= & \sum_{i=k}^{k+1} \mu_{i} l_{i} \\
= & \frac{a_{k+1}-a_{k}}{x_{k+1}-x_{k}} d_{*}^{2}+\frac{b_{k+1}-b_{k}}{x_{k+1}-x_{k}} d_{*} \\
& +\frac{x_{k+1} a_{k}-x_{k} a_{k+1}}{x_{k+1}-x_{k}} d_{*}+\frac{x_{k+1} b_{k}-x_{k} b_{k+1}}{x_{k+1}-x_{k}}
\end{aligned}
$$

Note that the output (30) is a quadratic function with respect to the input $d_{*}$. For simplification, $a_{k+1}=a_{k}=a$ with a constant $a$ is chosen so

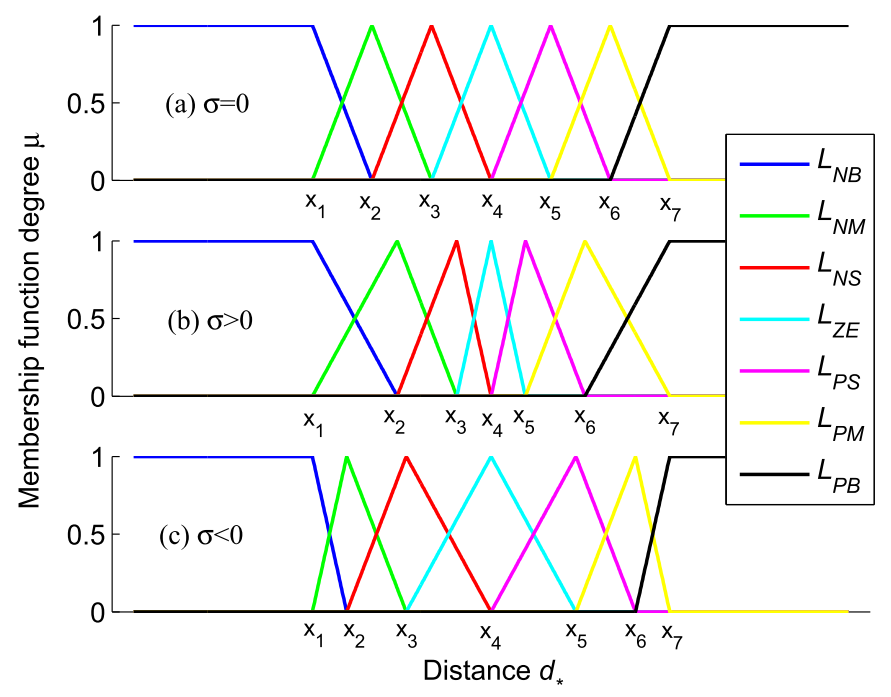

Fig. 4. Membership functions of the input $d_{*}$.
Table 2

Membership function of distance $d_{*}$.

\begin{tabular}{lll}
\hline Lines & Membership function & $x_{i}$ \\
\hline$L_{N B}$ & $\operatorname{trapmf}\left[-\infty,-\infty, x_{1}, x_{2}\right]$ & $x_{1}=-\frac{1+\lambda}{2 \sqrt{1+\lambda^{2}}}$ \\
$L_{N M}$ & $\operatorname{trimf}\left[x_{1}, x_{2}, x_{3}\right]$ & $x_{2}=-\frac{1+\lambda}{3 \sqrt{1+\lambda^{2}}}+\sigma$ \\
$L_{N S}$ & $\operatorname{trimf}\left[x_{2}, x_{3}, x_{4}\right]$ & $x_{3}=-\frac{1+\lambda}{6 \sqrt{1+\lambda^{2}}}+\sigma$ \\
$L_{Z E}$ & $\operatorname{trimf}\left[x_{3}, x_{4}, x_{5}\right]$ & $x_{4}=0$ \\
$L_{P S}$ & $\operatorname{trimf}\left[x_{4}, x_{5}, x_{6}\right]$ & $x_{5}=\frac{1+\lambda}{6 \sqrt{1+\lambda^{2}}}-\sigma$ \\
$L_{P M}$ & $\operatorname{trimf}\left[x_{5}, x_{6}, x_{7}\right]$ & $x_{6}=\frac{1+\lambda}{3 \sqrt{1+\lambda^{2}}}-\sigma$ \\
$L_{P B}$ & $\operatorname{trapmf}\left[x_{6}, x_{7}, \infty, \infty\right]$ & $x_{7}=\frac{1+\lambda}{2 \sqrt{1+\lambda^{2}}}$ \\
\hline
\end{tabular}

that this quadratic polynomial output is simplified into a linear function with respect to the input as

$f_{*}=\left(\frac{b_{k+1}-b_{k}}{x_{k+1}-x_{k}}+a\right) d_{*}+\frac{x_{k+1} b_{k}-x_{k} b_{k+1}}{x_{k+1}-x_{k}}$

On the other hand, $f_{*}=l_{i}$ when $d_{*} \notin\left(x_{1}, x_{7}\right)$ with $i \in\{1,7\}$. Hence, the output (28) can be rewritten as

$f_{*}=\kappa_{k} d_{*}+\gamma_{k}$

where the slope $\kappa_{k}$ and intercept $\gamma_{k}$ are defined in Table 3 .

According to (32), the simplified piecewise linear surface has a constant slope throughout the universe of discourse for an evenly arranged input and output membership functions. Obviously, a nonlinear control surface is more suitable to control an underactuated AUV with highly coupled dynamics and nonlinearity. Hence, the asymmetry factor $\sigma$ has been introduced in the membership function of the input as shown in Fig. 4. In addition, assume that all the intercepts in (27) are evenly arranged, namely $b_{k+1}-b_{k}=b$. In this case, the corresponding different slopes are listed in Table 4.

The nonlinear control surface in (32) is depicted in Fig. 5, which consists of eight linear control lines. Hence, the dynamics system under the nonlinear single-input fuzzy controller is regarded as a piecewise affine system composed of eight subsystems.

\subsubsection{Stability analysis}

For the $i$-th subsystem, substituting (26) into (2) leads to

$\left\{\begin{array}{l}\dot{u}=\dot{u}_{d}+\varsigma_{u} e_{u}+\int_{0}^{t}\left(l_{u} e_{u}+k_{f u} \gamma_{i}\right) d \tau+\frac{\tau_{E u}}{m_{11}} \\ \dot{q}=\dot{q}_{d}+\varsigma_{q} e_{q}+\int_{0}^{t}\left(l_{q} e_{q}+k_{f q} \gamma_{i}\right) d \tau+\frac{\tau_{E q}}{m_{55}} \\ \dot{r}=\dot{r}_{d}+\varsigma_{r} e_{r}+\int_{0}^{t}\left(l_{r} e_{r}+k_{f r} \gamma_{i}\right) d \tau+\frac{\tau_{E r}}{m_{66}}\end{array}\right.$

with $\varsigma_{*}=\frac{k_{f} k_{e_{*}} \kappa_{i} \lambda_{*}}{\sqrt{1+\lambda_{*}^{2}}}$ and $l_{*}=\frac{k_{f *} k_{e^{*}} \kappa_{i}}{\sqrt{1+\lambda_{*}^{2}}}$.

Let $s_{* 1}=\int_{0}^{t}\left(l_{*} e_{*}+k_{f *} \gamma_{i}\right) d \tau$. Then, (33) can be rewritten as

$\left\{\begin{array}{l}\ddot{s}_{u 1}+\varsigma_{u} \dot{s}_{u 1}+\iota_{u} s_{u 1}=d_{u} \\ \ddot{s}_{q 1}+\varsigma_{q} \dot{s}_{q 1}+\iota_{q} s_{q 1}=d_{q} \\ s_{r 1}+\varsigma_{r} \dot{s}_{r 1}+t_{r} s_{u 1}=d_{r}\end{array}\right.$

where $d_{*}=\varsigma_{*} k_{f^{*}} \gamma_{i}-l_{*} \frac{\tau_{E^{*}}}{m_{a}}$ with $a \in\{11,55,66\}$. Let $s_{* 2}=\dot{s}_{* 1}$ and define $s_{*}=\left[s_{* 1}, s_{* 2}\right]^{\top}$. Consider the following positive definite and radially

Table 3

Value of the slope $\kappa_{k}$ and the intercept $\gamma_{k}$.

\begin{tabular}{lll}
\hline$d_{*}$ & $\kappa_{k}$ & $\gamma_{k}$ \\
\hline$\left(-\infty, x_{1}\right]$ & $a$ & $b_{1}$ \\
$\left(x_{1}, x_{7}\right)$ & $\frac{b_{k+1}-b_{k}}{x_{k+1}-x_{k}}+a$ & $\frac{x_{k+1} b_{k}-x_{k} b_{k+1}}{x_{k+1}-x_{k}}$ \\
{$\left[x_{7}, \infty\right)$} & $a$ & $b_{7}$ \\
\hline
\end{tabular}


Table 4

Slope and intercept of fuzzy output $\left(b=\frac{1}{3}\right.$ and $\left.b_{1}=-1\right)$.

\begin{tabular}{lll}
\hline No. & $\kappa_{k}$ & $\gamma_{k}$ \\
\hline 0 & $\kappa_{0}=a$ & $\gamma_{0}=-1$ \\
1 & $\kappa_{1}=\frac{2 \sqrt{1+\lambda^{2}}}{1+\lambda+6 \sigma \sqrt{1+\lambda^{2}}}+a$ & $\gamma_{1}=\frac{-6 \sigma \sqrt{1+\lambda^{2}}}{1+\lambda+6 \sigma \sqrt{1+\lambda^{2}}}$ \\
2 & $\kappa_{2}=\frac{2 \sqrt{1+\lambda^{2}}}{1+\lambda}+a$ & $\gamma_{2}=\frac{-6 \sigma \sqrt{1+\lambda^{2}}}{1+\lambda}$ \\
3 & $\kappa_{3}=\frac{2 \sqrt{1+\lambda^{2}}}{1+\lambda-6 \sigma \sqrt{1+\lambda^{2}}}+a$ & $\gamma_{3}=0$ \\
4 & $\kappa_{4}=\frac{2 \sqrt{1+\lambda^{2}}}{1+\lambda-6 \sigma \sqrt{1+\lambda^{2}}}+a$ & $\gamma_{4}=0$ \\
5 & $\kappa_{5}=\frac{2 \sqrt{1+\lambda^{2}}}{1+\lambda}+a$ & $\gamma_{5}=\frac{6 \sigma \sqrt{1+\lambda^{2}}}{1+\lambda}$ \\
6 & $\kappa_{6}=\frac{2 \sqrt{1+\lambda^{2}}}{1+\lambda+6 \sigma \sqrt{1+\lambda^{2}}}+a$ & $\gamma_{6}=\frac{6 \sigma \sqrt{1+\lambda^{2}}}{1+\lambda+6 \sigma \sqrt{1+\lambda^{2}}}$ \\
7 & $\kappa_{7}=a$ & $\gamma_{7}=1$ \\
\hline
\end{tabular}

unbounded Lyapunov function candidate

$V=\frac{1}{2} e_{\text {ang }}^{\top} e_{\text {ang }}+s_{u}^{\top} P_{u} s_{u}+s_{q}^{\top} P_{q} s_{q}+s_{r}^{\top} P_{r} s_{r}$

where $P_{*}=\left[\begin{array}{cc}\frac{\varsigma_{*}+2 l_{*}}{2} & \frac{1}{2} \\ \frac{1}{2} & 1\end{array}\right]$ is a real symmetric positive definite matrix to

be chosen.

In view of (1), (21) and (34), the derivative of $V_{2}$ along the trajectories of the dynamics system is given by

$$
\begin{aligned}
& \dot{V}=e_{\theta}\left(\dot{\theta}_{d}-q\right)+e_{\psi}\left(\dot{\psi}_{d}-\frac{r}{\cos (\theta)}\right) \\
& +\left(s_{u}+2 l_{u}\right) s_{u 1} \dot{s}_{u 1}+\dot{s}_{u 1} s_{u 2}+s_{u 1} \dot{s}_{u 2}+2 s_{u 2} \dot{s}_{u 2} \\
& +\left(s_{q}+2 l_{q}\right) s_{q 1} \dot{s}_{q 1}+\dot{s}_{q 1} s_{q 2}+s_{q 1} \dot{s}_{q 2}+2 s_{q 2} \dot{s}_{q 2} \\
& +\left(\varsigma_{r}+2 l_{r}\right) s_{r 1} \dot{s}_{r 1}+\dot{s}_{r 1} s_{r 2}+s_{r 1} \dot{s}_{r 2}+2 s_{r 2} \dot{s}_{r 2} \\
& =-k_{\theta} e_{\theta}^{2}-\frac{k_{f q} \gamma_{i}}{l_{q}} e_{\theta}+\frac{1}{l_{q}} e_{\theta} s_{q 2} \\
& -k_{\psi} e_{\psi}^{2}-\frac{k_{f r} \gamma_{i}}{l_{r}} e_{\psi}+\frac{1}{l_{r}} e_{\theta} S_{r 2} \\
& -l_{u} s_{u 1}^{2}-\left(2 \varsigma_{u}-1\right) s_{u 2}^{2}+d_{u}\left(s_{u 1}+2 s_{u 2}\right) \\
& -l_{q} s_{q 1}^{2}-\left(2 \varsigma_{q}-1\right) s_{q 2}^{2}+d_{q}\left(s_{q 1}+2 s_{q 2}\right) \\
& -l_{r} s_{r 1}^{2}-\left(2 \varsigma_{r}-1\right) s_{r 2}^{2}+d_{r}\left(s_{r 1}+2 s_{r 2}\right) \\
& \leq-k_{\theta} e_{\theta}^{2}-\frac{k_{f q} \gamma_{i}}{l_{q}} e_{\theta}+\frac{1}{2 l_{q}} e_{\theta}^{2}+\frac{1}{2 l_{q}} s_{q 2}^{2} \\
& -k_{\psi} e_{\psi}^{2}-\frac{k_{f r} \gamma_{i}}{l_{r}} e_{\psi}+\frac{1}{2 l_{r}} e_{\psi}^{2}+\frac{1}{2 l_{r}} s_{r 2}^{2} \\
& -l_{u} s_{u 1}^{2}-\left(2 \varsigma_{u}-1\right) s_{u 2}^{2}+d_{u}\left(s_{u 1}+2 s_{u 2}\right) \\
& -l_{q} s_{q 1}^{2}-\left(2 \varsigma_{q}-1\right) s_{q 2}^{2}+d_{q}\left(s_{q 1}+2 s_{q 2}\right) \\
& -l_{r} s_{r 1}^{2}-\left(2 \varsigma_{r}-1\right) s_{r 2}^{2}+d_{r}\left(s_{r 1}+2 s_{r 2}\right) \\
& =-\left(k_{\theta}-\frac{1}{2 l_{q}}\right) e_{\theta}^{2}-\frac{k_{f q} \gamma_{i}}{l_{q}} e_{\theta} \\
& -\left(k_{\psi}-\frac{1}{2 l_{r}}\right) e_{\psi}^{2}-\frac{k_{f r} \gamma_{i}}{l_{r}} e_{\psi} \\
& -l_{u} s_{u 1}^{2}-\left(2 \varsigma_{u}-1\right) s_{u 2}^{2}+d_{u}\left(s_{u 1}+2 s_{u 2}\right) \\
& -l_{q} s_{q 1}^{2}-\left(2 \varsigma_{q}-1-\frac{1}{2 l_{q}}\right) s_{q 2}^{2}+d_{q}\left(s_{q 1}+2 s_{q 2}\right) \\
& -l_{r} s_{r 1}^{2}-\left(2 \varsigma_{r}-1-\frac{1}{2 l_{r}}\right) s_{r 2}^{2}+d_{r}\left(s_{r 1}+2 s_{r 2}\right)
\end{aligned}
$$

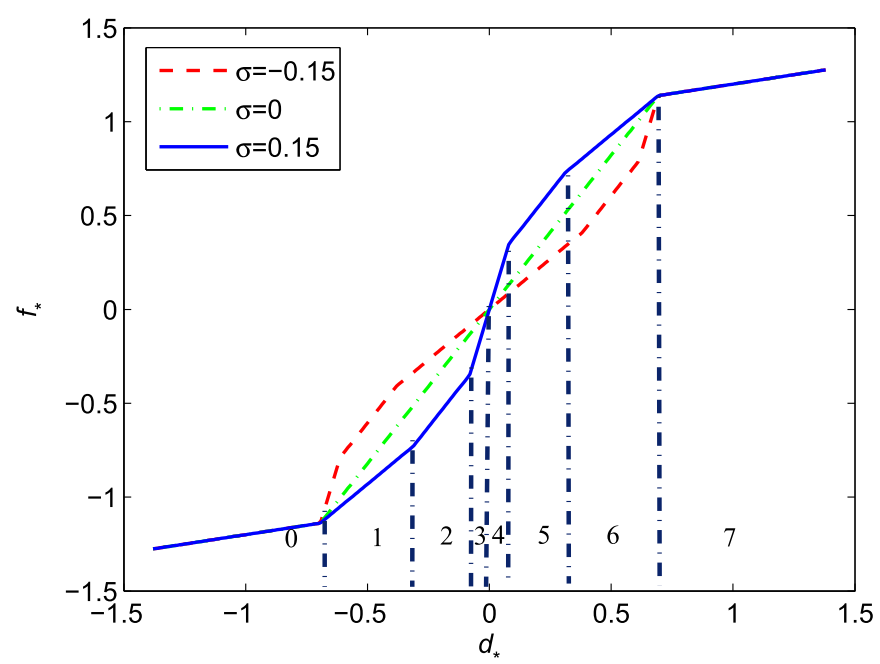

Fig. 5. Nonlinear control surface of the single-input fuzzy output.

Let $\quad k_{s 1}=\min \left\{k_{\theta}-\frac{1}{2 l_{q}}, k_{\psi}-\frac{1}{2 l_{r}}, l_{*}, 2 \varsigma_{*}-1-\frac{1}{2 l_{*}}\right\}>0 \quad$ and $\quad k_{s 2}=$ $2 \sqrt{2} \max \left\{\left|\frac{k_{f\left(\gamma_{i}\right.}}{\iota_{q}}\right|,\left|\frac{k_{f f} \gamma_{i}}{\iota_{r}}\right|, 2 d_{* \max }\right\}>0$ with $d_{* \max }=\left|\varsigma_{*} k_{f^{*}} \gamma_{i}\right|+l_{*} \frac{\tau_{E^{*} \max }}{m_{a}}$. Then, we obtain that

$$
\begin{aligned}
\dot{V} \leq & -k_{s 1}\left(e_{\theta}^{2}+e_{\psi}^{2}+s_{u 1}^{2}+s_{u 2}^{2}+s_{q 1}^{2}+s_{q 2}^{2}+s_{r 1}^{2}+s_{r 2}^{2}\right) \\
& +k_{s 2} \sqrt{e_{\theta}^{2}+e_{\psi}^{2}+s_{u 1}^{2}+s_{u 2}^{2}+s_{q 1}^{2}+s_{q 2}^{2}+s_{r 1}^{2}+s_{r 2}^{2}} \\
=- & \left(k_{s 1}-\delta\right)\left(e_{\theta}^{2}+e_{\psi}^{2}+s_{u 1}^{2}+s_{u 2}^{2}+s_{q 1}^{2}+s_{q 2}^{2}+s_{r 1}^{2}+s_{r 2}^{2}\right) \\
& -\delta\left(e_{\theta}^{2}+e_{\psi}^{2}+s_{u 1}^{2}+s_{u 2}^{2}+s_{q 1}^{2}+s_{q 2}^{2}+s_{r 1}^{2}+s_{r 2}^{2}\right) \\
& +k_{s 2} \sqrt{e_{\theta}^{2}+e_{\psi}^{2}+s_{u 1}^{2}+s_{u 2}^{2}+s_{q 1}^{2}+s_{q 2}^{2}+s_{r 1}^{2}+s_{r 2}^{2}}
\end{aligned}
$$

where $0<\delta<k_{s 1}$. Therefore,

$\dot{V} \leq-\left(k_{s 1}-\delta\right)\left(e_{\theta}^{2}+e_{\psi}^{2}+s_{u 1}^{2}+s_{u 2}^{2}+s_{q 1}^{2}+s_{q 2}^{2}+s_{r 1}^{2}+s_{r 2}^{2}\right)$

for $\sqrt{e_{\theta}^{2}+e_{\psi}^{2}+s_{u 1}^{2}+s_{u 2}^{2}+s_{q 1}^{2}+s_{q 2}^{2}+s_{r 1}^{2}+s_{r 2}^{2}} \geq \frac{k_{s 2}}{\delta}$. Further, we can conclude that the solutions $e_{\theta}, e_{\psi}, s_{u 1}, s_{u 2}, s_{q 1}, s_{q 2}, s_{r 1}$ and $s_{r 2}$ in (35) are uniformly ultimately bounded (Khalil, 1996). In addition, $\boldsymbol{e}_{*}$ is also uniformly ultimately bounded since $s_{* 2}=l_{*} e_{*}+k_{f^{*}} \gamma_{i}$ with constant $\iota_{*}, k_{f^{*}}, \gamma_{i}(* \in\{u, q, r\})$. In summary, the tracking errors in the dynamics system under the nonlinear single-input fuzzy controller are uniformly ultimately bounded.

\subsection{Sensitivity analysis}

In order to evaluate the performance of the nonlinear single-input fuzzy controller, the sensitivity analysis and comparison of the fuzzy controller responses are carried out by using different membership function distributions of the single input $d_{*}$ and slopes $\lambda_{*}$ in (25).

Three valid inputs in the vicinity of the equilibrium point and different membership function distributions of the single input are first chosen to analyze the sensitivity of the nonlinear single-input fuzzy controller as listed in Table 5. Obviously, the convergent distribution generates a larger control output than the other two distributions in the case of the same single input, which indicates that the convergent distribution has the potential to reject unknown environmental disturbances and guarantee better robustness.

Subsequently, different slopes in (25) are investigated and the corresponding fuzzy controller responses are listed in Table 6 . Through the separated analysis of each slope, it can be found that both of them give 
the same response characteristic as Table 5. In addition, the comparison of fuzzy controller responses among different slopes illustrates that a smaller slope can generate a larger control output. Hence, the sensitivity analysis and comparison of fuzzy controller response preliminary suggests that the combination of the convergent distribution and small slope for the single input have better robustness against external environmental disturbances.

\section{Case studies}

In order to illustrate the $3 \mathrm{D}$ path following performance of the designed framework as well as the nonlinear single-input fuzzy controller proposed in this paper, numerical simulations are carried out with an AUV dynamics model in Table 7, where all the parameters are given in Do and Pan (2009).

AUV paths are decomposed into multiple segments of straight lines and arcs. Thus, two primitive path types are considered in the following simulations: straight line and helical curve. In the straight line path following simulation, we focus on the sensitivity validation of the singleinput fuzzy controller and the choice of sensitivity gains. While in the helical curve path following simulation, we show the performance of the nonlinear single-input fuzzy controller with the convergent distribution and small slope for the single input in the presence of unknown environmental disturbances.

\subsection{Straight line path following}

In this case, assume that the AUV is exposed to the following constant environmental disturbances: $\tau_{E u}=-0.2 m_{11} d(t), \tau_{E v}=0.1 m_{22} d$ $(t), \tau_{E w}=-0.1 m_{33} d(t), \tau_{E q}=0.1 m_{55} d(t)$, and $\tau_{E r}=-0.2 m_{66} d(t)$ with

$d(t)=\left\{\begin{array}{c}1, t \in\left[t_{s}, t_{e}\right] \\ 0, t \notin\left[t_{s}, t_{e}\right]\end{array}\right.$

where $t_{s}$ and $t_{e}$ denote the starting time and vanishing time of environmental disturbances.

The desired straight line to be tracked by the AUV is parameterized by $x_{P}(\varpi)=\varpi, y_{P}(\varpi)=10$, and $z_{P}(\varpi)=50$ with $\varpi(0)=0$. The initial position and posture of the AUV are $[x(0), y(0), z(0), \theta(0), \psi(0)]=[5 \mathrm{~m}, 15 \mathrm{~m}, 55 \mathrm{~m}, 0 \mathrm{rad}, 0 \mathrm{rad}]$ and the initial speeds

$[u(0), v(0), w(0), q(0), r(0)]=[3 \mathrm{~m} / \mathrm{s}, 0 \mathrm{~m} / \mathrm{s}, 0 \mathrm{~m} / \mathrm{s}, 0 \mathrm{rad} / \mathrm{s}, 0 \mathrm{rad} / \mathrm{s}] . \quad$ The unknown environmental disturbances act on the AUV from 0s to $60 \mathrm{~s}$. The corresponding path following results under different membership function distributions are described in Fig. 6. Although all of them converge to the desired path, their performances are different from each other.

As shown in Fig. 7, the path following off-track error under the singleinput fuzzy controller with convergent distributions has the minimum drop when environmental disturbances vanish. In addition, the path following elevation angle error and azimuth angle error under the singleinput fuzzy controller with convergent distributions have the smoothest transition, the minimum drop and the shortest recovery time when environmental disturbances vanish. The specific values of response criteria considered are listed in Table 8, which quantitatively illustrates the nonlinear single-input fuzzy controller with convergent distributions has the best robustness against environmental disturbances.

The available control inputs including the surge force, pitch and yaw moments are given in Fig. 8, where they keep changing against external environmental disturbances. Note that the surge force and yaw moment under the single-input fuzzy controller with convergent distributions have the smoothest transitions against initial position and speed errors and external environmental disturbances.

The path following errors under the nonlinear single-input fuzzy controller with different slopes are described in Fig. 9. The specific values of response criteria considered are listed in Table 9, which quantitatively illustrates the nonlinear single-input fuzzy controller with a small slope
Table 5

Comparison of different distributions $\left(\lambda_{*}=0.5\right)$.

\begin{tabular}{lllll}
\hline No. & $d_{*}$ & $f_{*}(\sigma<0)$ & $f_{*}(\sigma=0)$ & $f_{*}(\sigma>0)$ \\
\hline 1 & -0.05 & -0.0546 & -0.0845 & -0.2364 \\
2 & 0 & 0 & 0 & 0 \\
3 & 0.05 & 0.0546 & 0.0845 & 0.2364 \\
\hline
\end{tabular}

Table 6

Comparison of different slopes $\left(\lambda_{*}=1\right.$ and $\left.\lambda_{*}=1.5\right)$.

\begin{tabular}{lllll}
\hline No. & $d_{*}$ & $f_{*}(\sigma<0)$ & $f_{*}(\sigma=0)$ & $f_{*}(\sigma>0)$ \\
\hline $1\left(\lambda_{*}=1\right)$ & -0.05 & -0.0532 & -0.0807 & -0.2045 \\
$2\left(\lambda_{*}=1\right)$ & 0 & 0 & 0 & 0 \\
$3\left(\lambda_{*}=1\right)$ & 0.05 & 0.0532 & 0.0807 & 0.2045 \\
$4\left(\lambda_{*}=1.5\right)$ & -0.05 & -0.0537 & -0.0821 & -0.2154 \\
$5\left(\lambda_{*}=1.5\right)$ & 0 & 0 & 0 & 0 \\
$6\left(\lambda_{*}=1.5\right)$ & 0.05 & 0.0537 & 0.0821 & 0.2154 \\
\hline
\end{tabular}

Table 7

AUV model parameters.

\begin{tabular}{lll}
\hline$G=10681 \mathrm{~N}$ & $h=0.0065 \mathrm{~m}$ & $m_{11}=1116 \mathrm{~kg}$ \\
\hline$d_{11}=25.5 \mathrm{~kg} \cdot \mathrm{s}^{-1}$ & $m_{22}=2133 \mathrm{~kg}$ & $d_{22}=138 \mathrm{~kg} \cdot \mathrm{s}^{-1}$ \\
$m_{33}=2133 \mathrm{~kg}$ & $d_{33}=138 \mathrm{~kg} \cdot \mathrm{s}^{-1}$ & $m_{55}=4061 \mathrm{~kg} \cdot \mathrm{m}^{2}$ \\
$d_{55}=490 \mathrm{~kg} \cdot \mathrm{m}^{2} \cdot \mathrm{s}^{-1}$ & $m_{66}=4061 \mathrm{~kg} \cdot \mathrm{m}^{2}$ & $d_{66}=490 \mathrm{~kg} \cdot \mathrm{m}^{2} \cdot \mathrm{s}^{-1}$ \\
\hline
\end{tabular}

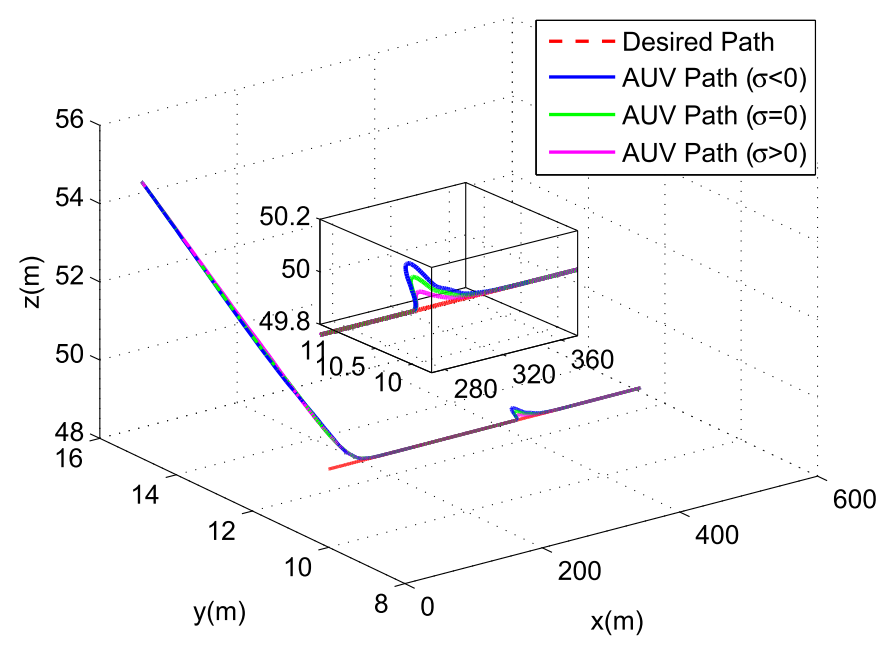

Fig. 6. 3D line path following.

has the best robustness against environmental disturbances.

The above numerical simulation results prove that the nonlinear single-input fuzzy controller with the combination of the convergent distribution and small slope for the single input has the best robustness against external disturbances. The next simulation case will illustrate the robust performance of this nonlinear single-input fuzzy controller in a more complicated helical curve path following mission.

\subsection{Helix path following}

In this case, assume that the AUV is exposed to the following timevarying environmental disturbances: $\tau_{E u}=-0.2 m_{11} d(t), \tau_{E v}=0.1 m_{22} d$ $(t), \tau_{E w}=-0.1 m_{33} d(t), \tau_{E q}=0.1 m_{55} d(t)$, and $\tau_{E r}=-0.2 m_{66} d(t)$ with $d(t)=1+0.1 \sin (0.2 t)$. Hence, while following a helical curve, the AUV is persistently exposed to the unknown environmental disturbances. The initial position and posture of the AUV are $[x(0), y(0), z(0), \theta(0), \psi(0)]=$ 

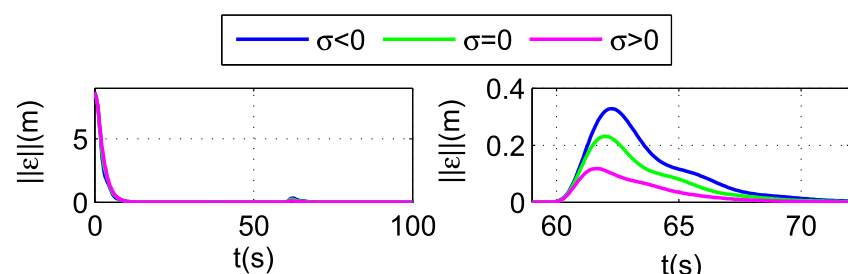

$\mathrm{t}(\mathrm{s})$
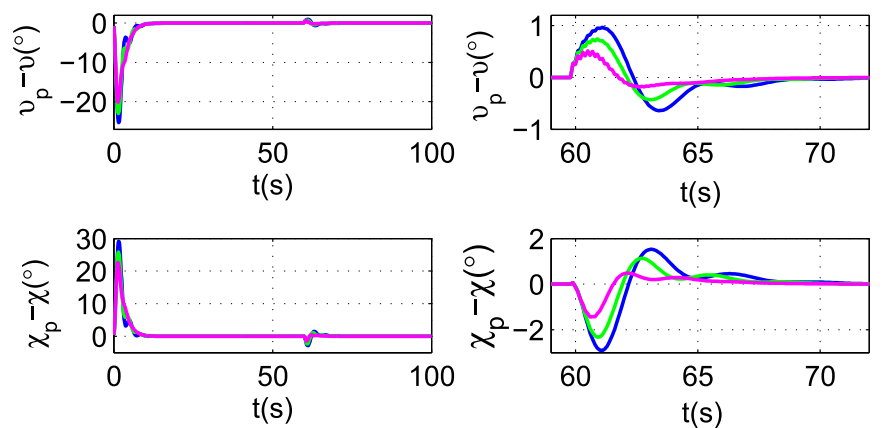

Fig. 7. 3D line path following errors under different distributions.

Table 8

3D line path following response criteria.

\begin{tabular}{llll}
\hline Item & $\sigma<0$ & $\sigma=0$ & $\sigma>0$ \\
\hline Off-track error drop & $0.3283 \mathrm{~m}$ & $0.2318 \mathrm{~m}$ & $0.1184 \mathrm{~m}$ \\
Elevation angle error drop & $0.9673^{\circ}$ & $0.741^{\circ}$ & $0.512^{\circ}$ \\
Elevation angle recovery time & $12.2 \mathrm{~s}$ & $11.4 \mathrm{~s}$ & $9.5 \mathrm{~s}$ \\
Azimuth angle error drop & $2.903^{\circ}$ & $2.318^{\circ}$ & $1.433^{\circ}$ \\
Azimuth angle recovery time & $14.2 \mathrm{~s}$ & $12.2 \mathrm{~s}$ & $10.7 \mathrm{~s}$ \\
\hline
\end{tabular}

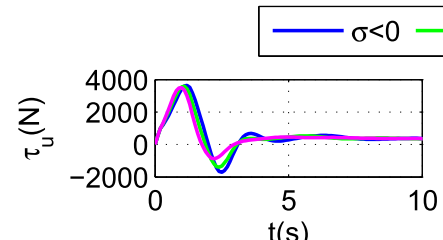

$t(s)$
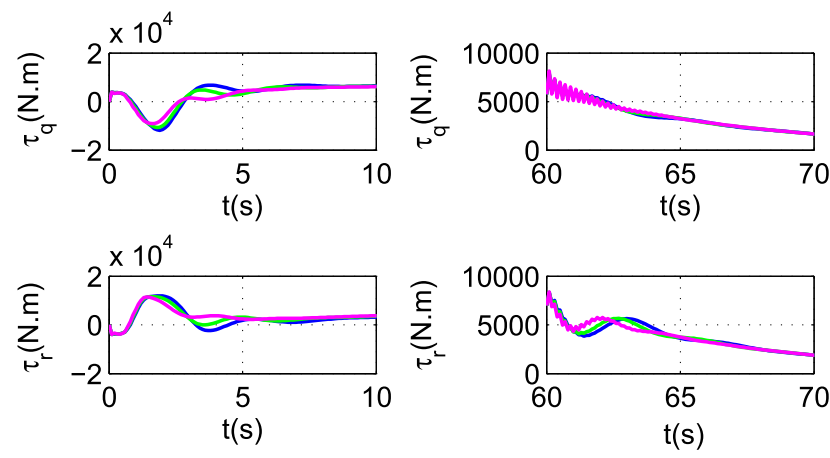

Fig. 8. 3D line path following control inputs.

$\left[90 \mathrm{~m},-5 \mathrm{~m}, 55 \mathrm{~m}, 0 \mathrm{rad}, \frac{3 \pi}{4} \mathrm{rad}\right]$ and the initial linear and angular speeds are $\quad[u(0), v(0), w(0), q(0), r(0)]=[3 \mathrm{~m} / \mathrm{s}, 0 \mathrm{~m} / \mathrm{s}, 0 \mathrm{~m} / \mathrm{s}, 0 \mathrm{rad} / \mathrm{s}, 0 \mathrm{rad} / \mathrm{s}]$. This helix path is parameterized by $x_{P}(\varpi)=80 \cos (0.2618 \varpi)$, $y_{P}(\varpi)=80 \sin (0.2618 \varpi)$, and $z_{P}(\varpi)=50-0.5 \varpi$ with $\varpi(0)=0$.

Note that $y_{P}$ and $z_{P}$ of the helix are time-varying instead of constant in the straight line, which places greater demands on the whole guidance and control system. The corresponding path following results are
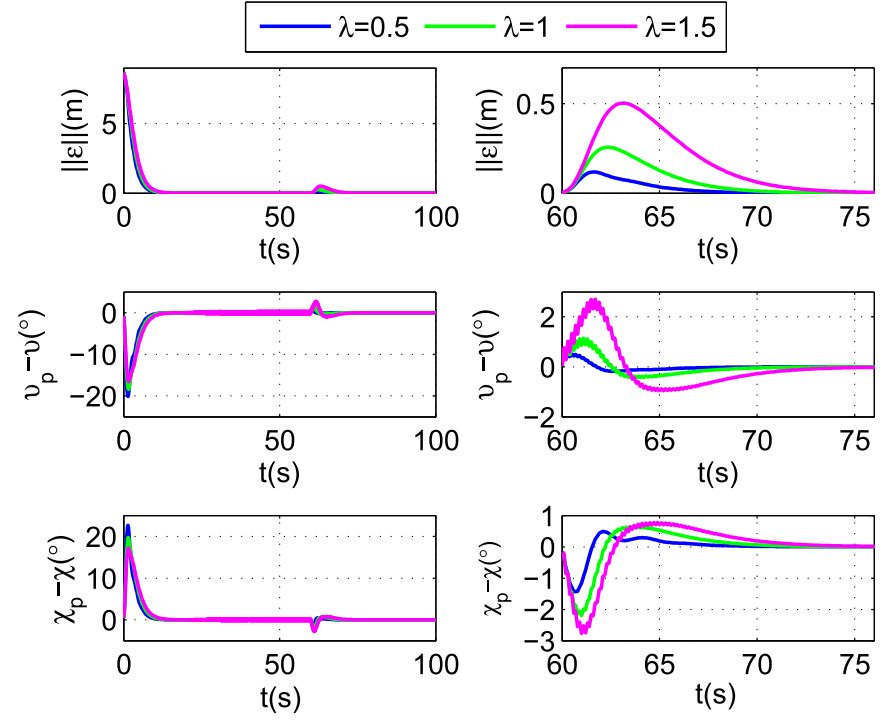

Fig. 9. 3D line path following errors under different slopes.

Table 9

3D line path following response criteria.

\begin{tabular}{llll}
\hline Item & $\lambda=1.5$ & $\lambda=1$ & $\lambda=0.5$ \\
\hline Off-track error drop & $0.5035 \mathrm{~m}$ & $0.2566 \mathrm{~m}$ & $0.1184 \mathrm{~m}$ \\
Elevation angle error drop & $2.708^{\circ}$ & $1.152^{\circ}$ & $0.512^{\circ}$ \\
Elevation angle recovery time & $18 \mathrm{~s}$ & $12 \mathrm{~s}$ & $8.5 \mathrm{~s}$ \\
Azimuth angle error drop & $2.764^{\circ}$ & $2.192^{\circ}$ & $1.422^{\circ}$ \\
Azimuth angle recovery time & $16.2 \mathrm{~s}$ & $13.1 \mathrm{~s}$ & $10.4 \mathrm{~s}$ \\
\hline
\end{tabular}

described in Figs. 10 and 11. As shown in Fig. 10, this AUV marked by a blue pentagram in $3 \mathrm{D}$ space converges to the virtual target point marked by a red circle, and finally follows the desired red path in the presence of unknown environmental disturbances. As shown in Fig. 11, all the position errors reduce to zero asymptotically and quickly. Moreover, the azimuth angle of the AUV in following a helix is non-zero and timevarying in the range of $-180^{\circ}$ to $180^{\circ}$. Yet, its elevation angle maintains at $1.35^{\circ}$ in the steady state. Despite of existing numerical differences, both the actual azimuth angle and elevation angle of the underactuated AUV converge to the respective desired profile in common, which indicates that the AUV exposed to unknown environmental disturbances follows the desired helix with its resultant speed aligned with the tangent of the spatial helix.

During the whole time domain, the linear and angular speeds are given in Fig. 12, where the underactuated AUV reaches a desired resultant speed of $4.5 \mathrm{~m} / \mathrm{s}$ but the sway speed and heave speed are non-zero. Hence, the underactuated AUV suffers from drift effects in sway and heave and transitions of the corresponding side-slip angle and angle of attack are shown in Fig. 12.

\section{Conclusion}

By designing an improved 3D guidance and nonlinear fuzzy control framework, this paper addresses the problem of coupled 3D path following for an underactuated AUV exposed to unknown environmental disturbances. The improved LOS guidance law transforms 3D path following position and orientation errors into controlled guidance speeds, by considering essentially equivalent coordinate transformation and underactuated property. The simplified and nonlinear single-input fuzzy controller is designed in the dynamics layer to track the guidance speeds with less computation cost and reject the unknown environmental 


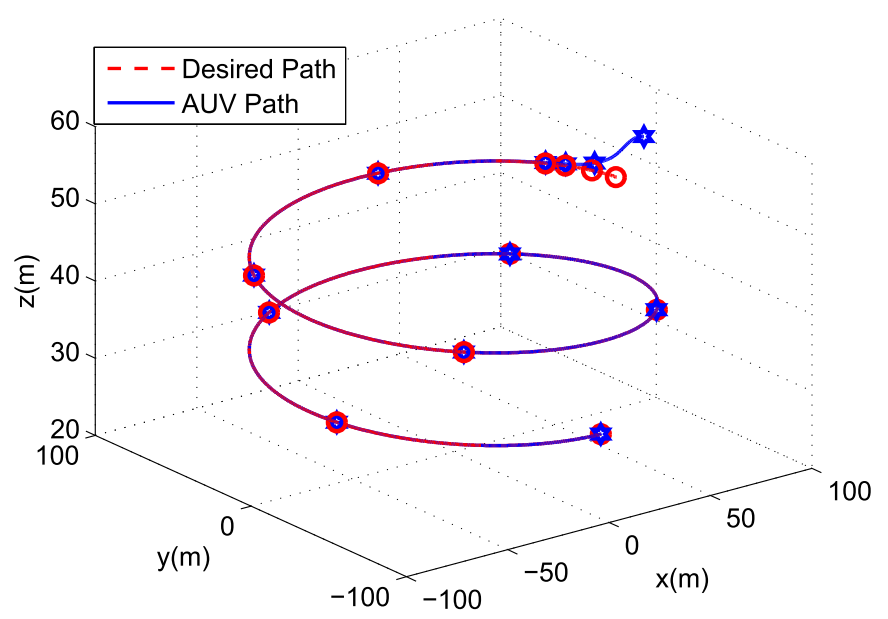

Fig. 10. 3D helix path following.
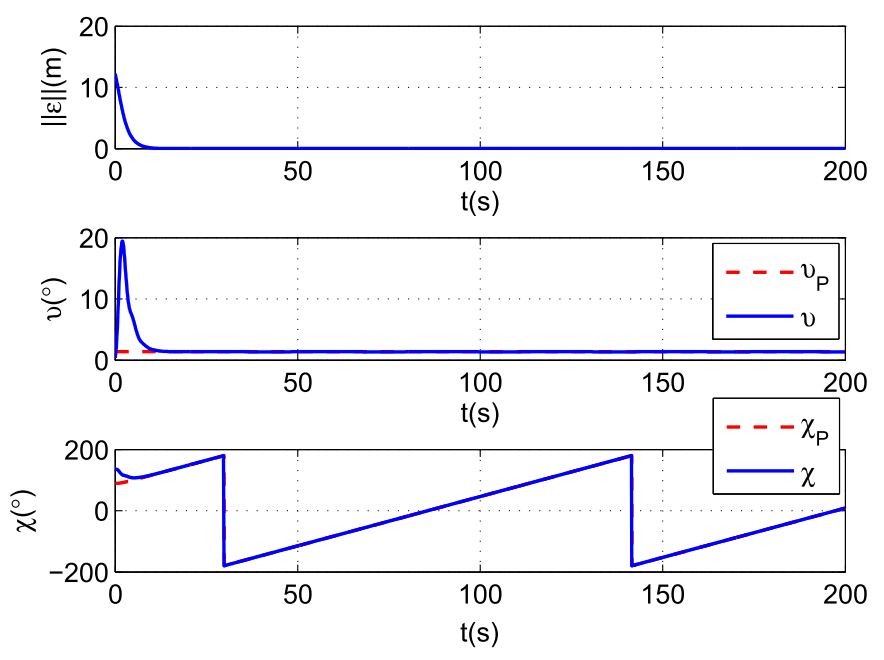

Fig. 11. 3D helix path following errors.
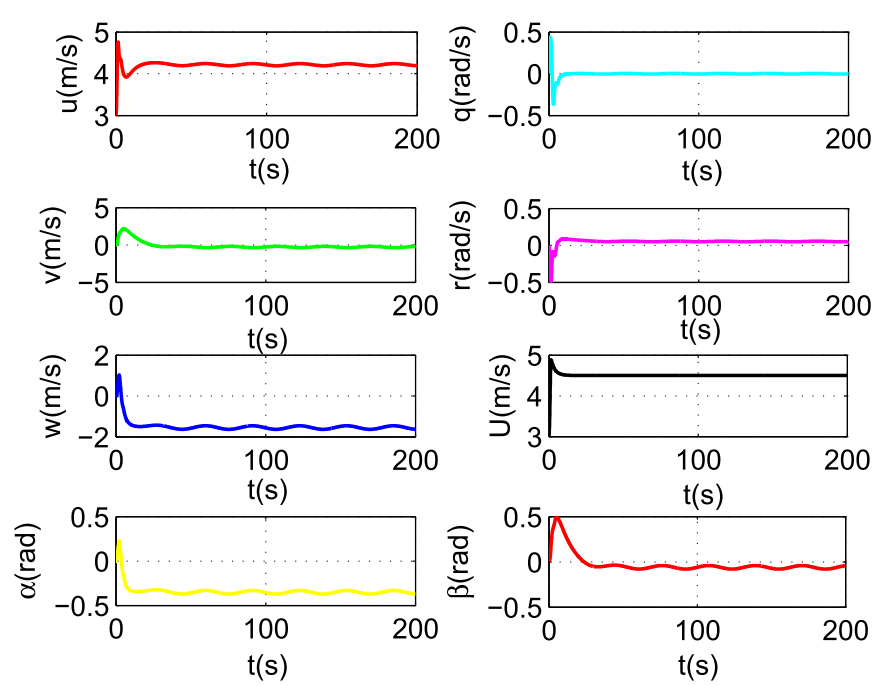

Fig. 12. The speeds and angles of the AUV.

disturbances in 3D path following. In addition, the sensitivity analysis suggests that the nonlinear fuzzy controller have better robustness against environmental disturbances than the linear fuzzy controller. Finally, numerical examples illustrate the robust path following performance of the proposed 3D guidance and nonlinear single-input fuzzy controller for an underactuated AUV. Future work can focus on testing the proposed 3D path following controller in open water using an AUV prototype.

\section{Acknowledgements}

This work is supported by National Natural Science Foundation of China (under Grant 51579111 and 51209100), the Fundamental Research Funds for the Central Universities (under Grant 2017KFYXJJ005), and State Key Laboratory of Ocean Engineering (under Grant 1504).

\section{References}

Aguiar, A.P., Hespanha, J.P., et al., 2007. Trajectory-tracking and path-following of underactuated autonomous vehicles with parametric modeling uncertainty. IEEE Trans. Autom. Control 52 (8), 1362-1379.

Breivik, M., Fossen, T., 2005. Principles of guidance-based path following in 2d and 3d. In: Proceedings of the 44th IEEE Conference on Decision and Control and 2005 European Control Conference (CDC-ECC'05), pp. 627-634.

Breivik, M., Fossen, T., 2005. Guidance-based path following for autonomous underwater vehicles. In: Proceedings of MTS/IEEE OCEANS, 2005, pp. 2807-2814.

Caharija, W., Pettersen, K.Y., Bibuli, M., Calado, P., Zereik, E., Braga, J., Gravdahl, J.T., Sorensen, A.J., Milovanovic, M., Bruzzone, G., 2016. Integral line-of-sight guidance and control of underactuated marine vehicles: theory, simulations, and experiments. IEEE Trans. Control Syst. Technol. 24, 1623-1642.

Chen, Y.Y., Tian, Y.P., 2015. Coordinated path following control of multi-unicycle formation motion around closed curves in a time-invariant flow. Nonlinear Dyn. 81 (1-2), 1005-1016.

Chen, W., Wei, Y., Zeng, J., Han, H., Jia, X., 2016. Adaptive terminal sliding mode ndobased control of underactuated auv in vertical plane. Discrete Dyn. Nat. Soc. 2016, $1-9$.

Choi, B.-J., Kwak, S.-W., Kim, B.K., 2000. Design and stability analysis of single-input fuzzy logic controller. IEEE Trans. Syst. Man, Cybern. Part B Cybern. 30 (2), 303-309.

Chu, Z., Zhu, D., Yang, S.X., 2017a. Observer-based adaptive neural network trajectory tracking control for remotely operated vehicle. IEEE Trans. Neural Netw. Learn. Syst. 28 (7), 1633-1645.

Chu, Z., Xiang, X., Zhu, D., Luo, C., Xie, D., 2017b. Adaptive fuzzy sliding mode diving control for autonomous underwater vehicle with input constraint. Int. J. Fuzzy Syst. https://doi.org/10.1007/s40815-017-0390-2.

Cui, R., Zhang, X., Cui, D., 2016. Adaptive sliding-mode attitude control for autonomous underwater vehicles with input nonlinearities. Ocean. Eng. 123, 45-54.

Do, K.D., 2016. Global robust adaptive path-tracking control of underactuated ships under stochastic disturbances. Ocean. Eng. 111, 267-278.

Do, K.D., Pan, J., 2009. Control of Ships and Underwater Vehicles: Design for Underactuated and Nonlinear Marine Systems. Springer Science \& Business Media.

Do, K., Pan, J., Jiang, Z., 2004. Robust and adaptive path following for underactuated autonomous underwater vehicles. Ocean. Eng. 31 (16), 1967-1997.

Encarnacao, P., Pascoal, A., 2000. 3d path following for autonomous underwater vehicle. In: Proceedings of the 39th IEEE Conference on Decision and Control, pp. 2977-2982.

Fossen, T.I., 2011. Handbook of Marine Craft Hydrodynamics and Motion Control. John Wiley \& Sons.

Fossen, T.I., Lekkas, A.M., 2017. Direct and indirect adaptive integral line-of-sight pathfollowing controllers for marine craft exposed to ocean currents. Int. J. Adapt. Control Signal Process. 31 (4), 445-463.

Fossen, T.I., Pettersen, K.Y., Galeazzi, R., 2015. Line-of-sight path following for dubins paths with adaptive sideslip compensation of drift forces. IEEE Trans. Control Syst. Technol. 23 (2), 820-827.

Gao, J., Proctor, A.A., Shi, Y., Bradley, C., 2016. Hierarchical model predictive imagebased visual servoing of underwater vehicles with adaptive neural network dynamic control. IEEE Trans. Cybern. 46 (10), 2323-2334.

Guo, J., Chiu, F.-C., Huang, C.-C., 2003. Design of a sliding mode fuzzy controller for the guidance and control of an autonomous underwater vehicle. Ocean. Eng. 30 (16), 2137-2155.

Ishaque, K., Abdullah, S.S., Ayob, S.M., Salam, Z., 2010. Single input fuzzy logic controller for unmanned underwater vehicle. J. Intell. Robotic Syst. 59 (1), 87-100.

Ishaque, K., Abdullah, S., Ayob, S., Salam, Z., 2011. A simplified approach to design fuzzy logic controller for an underwater vehicle. Ocean. Eng. 38 (1), 271-284.

Ju, Z., Liu, H., 2011. A unified fuzzy framework for human-hand motion recognition. IEEE Trans. Fuzzy Syst. 19 (5), 901-913.

Ju, Z., Liu, H., 2012. Fuzzy gaussian mixture models. Pattern Recognit. 45 (3), $1146-1158$.

Khalil, H.K., 1996. Nonlinear Systems. Prentice-Hall, New Jersey.

Lapierre, L., Soetanto, D., 2007. Nonlinear path-following control of an auv. Ocean. Eng. 34 (11-12), 1734-1744.

Liang, X., Qu, X., Hou, Y., Zhang, J., 2017. Three-dimensional path following control of underactuated autonomous underwater vehicle based on damping backstepping. Int. J. Adv. Robotic Syst. 14 (4), 1-9. 
Miao, J., Wang, S., Tomovic, M.M., Zhao, Z., 2017. Compound line-of-sight nonlinear path following control of underactuated marine vehicles exposed to wind, waves, and ocean currents. Nonlinear Dyn. 89 (4), 2441-2459.

Miao, J., Wang, S., Zhao, Z., Li, Y., Tomovic, M.M., 2017. Spatial curvilinear path following control of underactuated auv with multiple uncertainties. ISA Trans. 67, 107-130.

Peng, Z., Wang, D., Chen, Z., Hu, X., Lan, W., 2013. Adaptive dynamic surface control for formations of autonomous surface vehicles with uncertain dynamics. IEEE Trans. Control Syst. Technol. 21 (2), 513-520.

Peng, Z., Wang, D., Shi, Y., Wang, H., Wang, W., 2015. Containment control of networked autonomous underwater vehicles with model uncertainty and ocean disturbances guided by multiple leaders. Inf. Sci. 316, 163-179.

Peng, Z., Wang, J., Wang, D., 2017. Distributed containment maneuvering of multiple marine vessels via neurodynamics-based output feedback. IEEE Trans. Ind. Electron. 64 (5), 3831-3839.

Peng, Z., Wang, J., Wang, D., 2017. Distributed maneuvering of autonomous surface vehicles based on neurodynamic optimization and fuzzy approximation. IEEE Trans. Control Syst. Technol. https://doi.org/10.1109/TCST.2017.2699167.

Peymani, E., Fossen, T.I., 2015. Path following of underwater robots using lagrange multipliers. Robot. Aut. Syst. 67, 44-52.

Qiao, L., Zhang, W., 2017. Adaptive nonsingular integral terminal sliding mode tracking control for autonomous underwater vehicles. IET Control Theory \& Appl. 11 (8), 1293-1306.

Shojaei, K., Dolatshahi, M., 2017. Line-of-sight target tracking control of underactuated autonomous underwater vehicles. Ocean. Eng. 133, 244-252.

Tanakitkorn, K., Wilson, P.A., Turnock, S.R., Phillips, A.B., 2017. Depth control for an over-actuated, hover-capable autonomous underwater vehicle with experimental verification. Mechatronics 41, 67-81.

Wang, Y., Zhang, M., Wilson, P.A., Liu, X., 2015. Adaptive neural network-based backstepping fault tolerant control for underwater vehicles with thruster fault. Ocean. Eng. 110, 15-24.

Wang, N., Er, M.J., Sun, J.C., Liu, Y.C., 2016. Adaptive robust online constructive fuzzy control of a complex surface vehicle system. IEEE Trans. Cybern. 46 (7), 1511-1523.

Wang, N., Sun, J.C., Er, M.J., 2017. Tracking-error-based universal adaptive fuzzy control for output tracking of nonlinear systems with completely unknown dynamics. IEEE Trans. Fuzzy Syst. https://doi.org/10.1109/TFUZZ.2017.2697399.

Wang, N., Su, S.F., Yin, J., Zheng, Z., Er, M.J., 2017. Global asymptotic model-free trajectory-independent tracking control of an uncertain marine vehicle: an adaptive universe-based fuzzy control approach. IEEE Trans. Fuzzy Syst. https://doi.org/ 10.1109/TFUZZ.2017.2737405.

Wang, N., Qian, C., Sun, Z.-Y., 2017. Global asymptotic output tracking of nonlinear second-order systems with power integrators. Automatica 80, 156-161.

Xiang, X., Jouvencel, B., Parodi, O., 2010. Coordinated formation control of multiple autonomous underwater vehicles for pipeline inspection. Int. J. Adv. Robotic Syst. 7 (1), 75-84.
Xiang, X., Yu, C., Zheng, J., Xu, G., 2015. Motion forecast of intelligent underwater sampling apparatus-part i: design and algorithm. Indian J. Geo-Marine Sci. 44 (12), 1962-1970.

Xiang, X., Lapierre, L., Jouvencel, B., 2015. Smooth transition of auv motion control: from fully-actuated to under-actuated configuration. Robot. Aut. Syst. 67, 14-22.

Xiang, X., Yu, C., Zhang, Q., Xu, G., 2016. Path-following control of an auv: fully actuated versus under-actuated configuration. Mar. Technol. Soc. J. 50 (1), 34-47.

Xiang, X., Yu, C., Niu, Z., Zhang, Q., 2016. Subsea cable tracking by autonomous underwater vehicle with magnetic sensing guidance. Sensors 16 (8), 1335.

Xiang, X., Yu, C., Zhang, Q., 2017. Robust fuzzy 3d path following for autonomous underwater vehicle subject to uncertainties. Comput. Oper. Res. 84, 165-177.

Xiang, X., Yu, C., Zhang, Q., 2017. On intelligent risk analysis and critical decision of underwater robotic vehicle. Ocean. Eng. 140, 453-465.

Xiang, X., Yu, C., Lapierre, L., Zhang, J., Zhang, Q., 2017c. Survey on fuzzy-logic-based guidance and control of marine surface vehicles and underwater vehicles. Int. J. Fuzzy Syst. In press.

Xiang, X., Liu, C., Su, H., Zhang, Q., 2017d. On decentralized adaptive full-order sliding mode control of multiple UAVs. ISA Trans. https://doi.org/10.1016/ j.isatra.2017.09.008.

Yi, B., Qiao, L., Zhang, W., 2016. Two-time scale path following of underactuated marine surface vessels: design and stability analysis using singular perturbation methods. Ocean. Eng. 124, 287-297.

Yu, C., Xiang, X., Zhang, Q., Xu, G., 2017. Adaptive fuzzy trajectory tracking control of an under-actuated autonomous underwater vehicle subject to actuator saturation. Int. J. Fuzzy Syst. https://doi.org/10.1007/s40815-017-0396-9.

Zhang, F., Fratantoni, D.M., Paley, D.A., Lund, J.M., Leonard, N.E., 2007. Control of coordinated patterns for ocean sampling. Int. J. Control 80 (7), 1186-1199.

Zhang, Q., Lapierre, L., Xiang, X., 2013. Distributed control of coordinated path tracking for networked nonholonomic mobile vehicles. IEEE Trans. Ind. Inf. 9 (1), 472-484.

Zhang, F., Marani, G., Smith, R.N., Choi, H.T., 2015. Future trends in marine robotics. IEEE Robot. Autom. Mag. 22 (1), 14-21, 122.

Zheng, Z., Sun, L., Xie, L., 2017. Error-constrained los path following of a surface vessel with actuator saturation and faults. IEEE Trans. Syst. Man, Cybern. Syst. https:// doi.org/10.1109/TSMC.2017.2717850.

Zheng, Z., Huang, Y., Xie, L., Zhu, B., 2017. Adaptive trajectory tracking control of a fully actuated surface vessel with asymmetrically constrained input and output. IEEE Trans. Control Syst. Technol. https://doi.org/10.1109/TCST.2017.2728518.

Zheng, Z., Feroskhan, M., 2017c. Path following of a surface vessel with prescribed performance in the presence of input saturation and external disturbances. IEEE/ ASME Trans. Mech. https://doi.org/10.1109/TMECH.2017.2756110.

Zhu, D., Cao, X., Sun, B., Luo, C., 2017. Biologically inspired self-organizing map applied to task assignment and path planning of an auv system. IEEE Trans. Cognitive Dev. Syst. https://doi.org/10.1109/TCDS.2017.2727678. 